\title{
Degenerate elliptic equations for resonant wave problems*
}

\author{
A. Nicolopoulos† M. Campos Pinto; B. Després ${ }^{\S}$ and P. Ciarlet Jr.ף
}

July 16, 2019

\begin{abstract}
The modeling of resonant waves in $2 \mathrm{D}$ plasma leads to the coupling of two degenerate elliptic equations with a smooth coefficient $\alpha$ and compact terms. The coefficient $\alpha$ changes sign. The region where $\{\alpha>0\}$ is propagative, and the region where $\{\alpha<0\}$ is non propagative and elliptic. The two models are coupled through the line $\Sigma=\{\alpha=0\}$. Generically, it is an ill-posed problem, and additional information must be introduced to get a satisfactory treatment at $\Sigma$. In this work we define the solution by relying on the limit absorption principle $\left(\alpha\right.$ is replaced by $\left.\alpha+i 0^{+}\right)$in an adapted functional setting. This setting lies on the decomposition of the solution in a regular part and a singular part, which originates at $\Sigma$, and on quasi-solutions. It leads to a new well-posed mixed variational formulation with coupling. As we design explicit quasi-solutions, numerical experiments can be carried out, which illustrate the good properties of this new tool for numerical computation.
\end{abstract}

Keywords: degenerate elliptic equations, weighted Sobolev spaces, singular solutions, manufactured solutions, mixed variational formulations

\section{Introduction}

Resonant waves appear in various electromagnetic phenomenons, such as: fusion plasma heating where a wave is sent inside a plasma and transfers energy to the particles in a localized region [9, 7, 19]; cloaking devices where the transition between metamaterials of negative index and non-dissipative dielectrics is exploited [1, 6, 17; ; or photoacoustic imaging of biological tissues where metallic nanoparticles' heating 22 is used. The model problem for resonant waves throughout this work is the degenerate equation, elliptic where $\alpha<0$,

$$
\left\{\begin{aligned}
-\operatorname{div}(\alpha \nabla u)-u & =0 \quad \text { in } \Omega \subset \mathbb{R}^{2} \\
\alpha \partial_{n} u+i \lambda u & =f \quad \text { on } \Gamma
\end{aligned}\right.
$$

where $\lambda>0$ is a positive scalar and $f \in L^{2}(\Gamma)$ is complex valued on the boundary $\Gamma=\partial \Omega$. The resonance occurs at the transition between the propagative and non propagative regions of the domain. It is characterized by a coefficient $\alpha \in \mathcal{C}^{2}(\bar{\Omega})$ that changes sign inside the domain $\Omega$, typically over a closed curve denoted as $\Sigma$. In this study, $\Sigma \neq \emptyset$ is totally enclosed in the domain $\Omega$, and $\alpha$ behaves as a signed distance to $\Sigma=\{\alpha=0\}$. The equation (1.1) can be seen as two separate degenerate ellipitic equations, one based in $\{\alpha<0\}$, the other in $\{\alpha>0\}$ with remaining compact terms, both coupled at $\Sigma$.

This model is easily obtained from the time harmonic Maxwell's equations in fusion magnetized plasma, which are often used to model plasma heating in a tokamak [21, 16. In this context, the system of PDEs is

$$
\left\{\begin{array}{r}
\operatorname{curl} B-\epsilon \mathbf{E}=0 \\
B-\operatorname{curl} \mathbf{E}=0
\end{array}\right.
$$

where $\underline{\underline{\epsilon}}$ is the so-called cold plasma permittivity tensor, which encodes the specific propagation properties of the magnetized plasma. It is of the form

$$
\underline{\underline{\epsilon}}=\left(\begin{array}{cc}
\alpha & i \delta \\
-i \delta & \alpha
\end{array}\right)
$$

\footnotetext{
${ }^{*}$ This work has been carried out within the framework of the EUROfusion Consortium and has received funding from the European research and training programme under grant agreement N 633053. The views and opinions expressed herein do not necessarily reflect those of the European Commission.

${ }^{\dagger}$ LJLL, Sorbonne Université, 4 place Jussieu, F-75005 Paris, France

$\ddagger$ CNRS and LJLL, Sorbonne Université, 4 place Jussieu, F-75005 Paris, France

§IUF and LJLL, Sorbonne Université, 4 place Jussieu, F-75005 Paris, France

๑POEMS, ENSTA Paris, Institut Polytechnique de Paris, 828 boulevard des Maréchaux, F-91762 Palaiseau, France
} 
with real coefficients that depend on the constant frequency $\omega>0$ of the wave sent into the plasma, and on the space variable $\mathbf{x} \in \Omega$ through the plasma parameters. Precisely,

$$
\alpha(\mathbf{x})=\left(\frac{\omega}{c}\right)^{2}\left(1-\frac{\omega_{p}^{2}(\mathbf{x})}{\omega^{2}-\omega_{c}^{2}}\right) \in \mathbb{R}, \quad \delta(\mathbf{x})=\left(\frac{\omega}{c}\right)^{2}\left(\frac{\omega_{c} \omega_{p}^{2}(\mathbf{x})}{\omega\left(\omega^{2}-\omega_{c}^{2}\right)}\right) \in \mathbb{R} .
$$

Here $\omega_{p}$ is the plasma pulsation. It varies in space and corresponds to the frequency of oscillations of slightly perturbed electrons as they return to equilibrium. And $\omega_{c}$ is the cyclotron pulsation, the frequency to which electrons gyrate around the magnetic field. We are interested in the singular behavior of the upper hybrid resonance, localized at $\Sigma=\left\{\mathbf{x} \in \mathbb{R}^{2}, \omega^{2}=\omega_{p}^{2}(\mathbf{x})+\omega_{c}^{2}\right\}=\left\{\mathbf{x} \in \mathbb{R}^{2}, \alpha(\mathbf{x})=0\right\}$. We assume that $\Sigma$ is a closed curve that separates the domain $\Omega$ in two. On $\Sigma$, the off-diagonal coefficient is proportional to $\delta=\frac{\omega \omega_{c}}{c^{2}}>0$. We thus consider $\delta$ to be positive and bounded below by a non-zero constant. For simplicity, we will consider from now on that $\delta$ is constant. In problem (1.2), the normal component of the electric field is expected [7, 19] to have a singularity at $\Sigma$ of the order of $1 / \alpha$. This singularity does not belong to $L^{2}(\Omega)$, nor to $L^{1}(\Omega)$. Conclusions drawn from the $1 \mathrm{D}$ case led us to consider the auxiliary fields $\tilde{\mathbf{E}}:=\mathbf{E}-\nabla \frac{B}{i \delta}$ and $u:=\frac{B}{i \delta}$. The field $\tilde{\mathbf{E}}$ is the so-called regular part of the electric field [2, Chap. 6]. Developing the algebra, it yields $i \delta \operatorname{curl} u+i \delta \underline{\underline{\epsilon}} \nabla u=\alpha \nabla u$. One gets

$$
\left\{\begin{aligned}
\alpha \nabla u-\underline{\epsilon} \tilde{\mathbf{E}} & =0 \\
i \delta u-\operatorname{curl} \tilde{\mathbf{E}} & =0
\end{aligned}\right.
$$

Reformulated on the unknown $u$, this gives $\operatorname{curl}\left(\alpha \underline{\underline{\epsilon}}^{-1} \nabla u\right)-i \delta u=0$, where

$$
\underline{\underline{\epsilon}}^{-1}=\frac{1}{\alpha^{2}-\delta^{2}}\left(\begin{array}{cc}
\alpha & -i \delta \\
i \delta & \alpha
\end{array}\right)=-\sum_{n \geq 0} \frac{\alpha^{2 n+1}}{\delta^{2 n+2}}\left(\begin{array}{cc}
1 & 0 \\
0 & 1
\end{array}\right)+\sum_{n \geq 0} \frac{\alpha^{2 n}}{\delta^{2 n+1}}\left(\begin{array}{cc}
0 & i \\
-i & 0
\end{array}\right),
$$

which yields the expansion

$$
-\frac{1}{\delta^{2}} \operatorname{div}(\alpha \nabla u)-\sum_{n \geq 0} \operatorname{div}\left(\frac{\alpha^{2 n+2}}{\delta^{2 n+3}}\left(\begin{array}{cc}
\alpha / \delta & i \\
-i & \alpha / \delta
\end{array}\right) \nabla u\right)-u=0 .
$$

Given that $\alpha$ vanishes on $\Sigma$, the terms under the summation sign can be neglected as they are factors of $\alpha^{2 n+2}$ with $n \geq 0$. Adding mixed boundary conditions, it comes down to

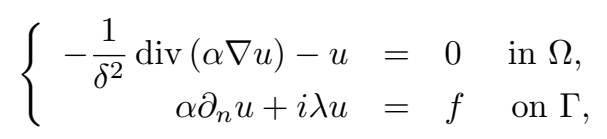

which is the model problem (1.1) for $\delta=1$. We chose $\alpha$ to behave as a signed distance to $\Sigma$.

The main mathematical consequence of the degeneracy of $\alpha$ at $\Sigma$ is that singular solutions localized at $\Sigma$ may occur.

Similar equations and problems arise from the study of metamaterials as stated previously. It is shown in [6, 4, 5, that the Fredholm well-posedness of the problem depends on the contrast between the negative permittivity of the metamaterial and the positive permittivity of the dielectric, as well as the geometry of the interface. The metamaterial problem was studied under a limiting absorption principle point of view in combination with Agmon-Douglis-Niremberg elliptic a priori estimates in [17. However the permittivity is constant on each side and does not vanish at the interface, so that the situation is different for hybrid resonances in fusion plasma.

In strict terms of partial differential equations, (1.1) is a coupling of two degenerate equations of different signs. There is ellipticity in the non propagative region, and ellipticity up to the addition of compact terms in the propagative region. In each separate case, the degeneracy concerns a part of the border. Both are then coupled through the degenerate border. The class $\mathcal{M}$ of Muckenhoupt weights, made of coefficients which are locally integrable as well as their inverse [23, is commonly used to study degenerate elliptic equations and we refer to [13, 12] for results from the elliptic community. Unfortunately, $\operatorname{dist}_{\Sigma} \notin \mathcal{M}$. To the best of our knowledge, the coupling of two degenerate elliptic equations with a coefficient proportional to the distance to the subset of the border, as $|\alpha|=\mid$ dist $_{\Sigma} \mid$, is not treated in the literature.

A classical mean to recover the correct solution of such time harmonic wave equations is to resort to a limiting absorption principle. In this work, as in previous 1D works [7, 19], a small parameter $\nu>0$ 
is introduced, which regularizes the equation and corresponds to a negligible physical quantity. The regularized problem is then

$$
\left\{\begin{array}{rlll}
-\operatorname{div}\left((\alpha+i \nu) \nabla u^{\nu}\right)-u^{\nu} & = & 0 & \text { in } \Omega \\
(\alpha+i \nu) \partial_{n} u^{\nu}+i \lambda u^{\nu} & = & f & \text { on } \Gamma
\end{array}\right.
$$

Since 1.3 is well-posed for $\nu>0$ according to the Lax-Milgram theorem, the whole point is to find a way to pass to the limit as $\nu \rightarrow 0^{+}$to construct a solution to 1.1 .

Our contribution in this work is to propose an original variational formulation for the limit problem $\nu=0^{+}$. The mathematical idea is to use a domain decomposition approach. Physically, we decouple the equations in the propagative region $\{\alpha>0\}$ from the equations in the non propagative region $\{\alpha<0\}$, and focus on the transmission area. Equations 1.1 and 1.3 thus separate into two similar equations written respectively in subdomains $\Omega_{1}=\{\mathbf{x} \in \Omega, \alpha(\mathbf{x})>0\}$ and $\Omega_{2}=\{\mathbf{x} \in \Omega, \alpha(\mathbf{x})<0\}$. The main difficulty consists in finding transmission conditions on $\Sigma=\partial \Omega_{1} \cap \partial \Omega_{2}=\{\mathbf{x} \in \Omega, \alpha(\mathbf{x})=0\}$.

To adress these questions, the method that we propose is based on a novel characterization of the singular behavior on $\Sigma$ of the solution, with the design of complex logarithmic quasi-solutions in combination with an adapted functional apparatus. Before stating the main results of this work, we develop the type of singular solutions we have in mind on a simple explicit solution in dimension one. The main ideas behind the construction will appear through this example: we will see that although a generic singular solution has no Dirichlet trace at the singular locus $\Sigma$, it remains possible to define a Neumann type trace for the flux at $\Sigma$.

\subsection{A singular solution in 1D and hint of the functional setting}

In dimension one with a coefficient $\alpha(x)=x$, the model problem 1.1 writes in a simpler form

$$
-\left(x u^{\prime}(x)\right)^{\prime}-u(x)=0, \quad \forall x \in \Omega=(-1,1) .
$$

The propagative region is $\Omega_{1}=\{0<x<1\}$. The non propagative region is $\Omega_{2}=\{-1<x<0\}$. We first describe the analytical solutions to $(1.4)$ and the singularity occuring at $\Sigma=\{0\}$. Then we motivate a convenient weighted functional setting that is adapted to our representation of the solutions.

One can check that $v(x)=u\left(x^{2} / 2\right)$ verifies the Bessel equation of order 0 . So $v$ is a linear combination of the Bessel functions of order 0 ,

$$
J_{0}: z \in \mathbb{C} \mapsto \sum_{k \geq 0} \frac{(-1)^{k}}{(k !)^{2}}\left(\frac{z^{2}}{4}\right)^{k} \quad \text { and } \quad Y_{0}: z \in \mathbb{C}^{*} \mapsto \frac{2}{\pi}\left(\left(\log \frac{z}{2}+\gamma\right) J_{0}(z)+\sum_{k \geq 1} \frac{(-1)^{k+1} H_{k}}{(k !)^{2}}\left(\frac{z^{2}}{4}\right)^{k}\right)
$$

where $\gamma$ is the Euler constant and $H_{k}$ the harmonic sum of order $k$, see [20] for more details on these special functions. A solution $u$ of 1.4 is a linear combination of $J_{0}(2 \sqrt{\cdot})$ and $Y_{0}(2 \sqrt{\cdot})$. But since equation (1.4) degenerates at $\Sigma$, no natural or immediate continuity relations can be deduced at $\Sigma$. In consequence, $u$ is a linear combination of $J_{0}(2 \sqrt{\cdot})$ and $Y_{0}(2 \sqrt{\cdot})$ separately on $\Omega_{1}$ and $\Omega_{2}$. A specific study is needed at $\Sigma$. Also, since $Y_{0}$ has a logarithmic singularity, so does a generic solution $u$, separately in $\Omega_{1}$ and $\Omega_{2}$. Once again, this singularity inhibits continuity of $u$ or $u^{\prime}$ at $x=0$. Nevertheless, a solution to (1.4) with the minimal requirement that $u \in L_{\mathrm{loc}}^{2}(\Omega)$ satisfies $x u^{\prime} \in C^{0}(\Omega)$. In particular, the continuous function $x u^{\prime}$ has a trace at 0 , which may be zero or non zero. This information is of tremendous importance in the rest of this work.

In order to exploit this idea, we introduce the spaces $H_{1 / 2}^{1}\left(\Omega_{j}\right):=\left\{v \in L^{2}\left(\Omega_{j}\right), \int_{\Omega_{j}}|x|\left|v^{\prime}(x)\right|^{2} \mathrm{~d} x<\infty\right\}$ for $j=1,2$. These spaces arise when integrating $(1.4)$ by parts, and are natural in the variational formulation of this problem. We next observe that a function $u_{j} \in H_{1 / 2}^{1}\left(\Omega_{j}\right)$ that verifies weakly equation (1.4) on $\Omega_{j}$ is such that $x u_{j}^{\prime} \in H^{1}\left(\Omega_{j}\right)$. Therefore one can write

$$
\left.x u_{j}^{\prime}(x)\right|_{x=0}=\lim _{\epsilon \rightarrow 0} \frac{1}{\epsilon} \int_{\Omega_{j} \cap\{|x|<\epsilon\}} x u_{j}^{\prime}(x) \mathrm{d} x,
$$

so that the trace of $x u_{j}^{\prime}$ at $\Sigma$ has a meaning. Indeed, since $\sqrt{x} u_{j}^{\prime} \in L^{2}\left(\Omega_{j}\right)$, a Cauchy-Schwarz inequality and the dominated convergence theorem lead to

$$
\frac{1}{\epsilon} \int_{\Omega_{j} \cap\{|x|<\epsilon\}}\left|x u_{j}^{\prime}(x)\right| \mathrm{d} x \leq \frac{1}{\sqrt{2}}\left(\int_{\Omega_{j} \cap\{|x|<\epsilon\}}|x|\left|u_{j}^{\prime}(x)\right|^{2} \mathrm{~d} x\right)^{1 / 2} \underset{\epsilon \rightarrow 0}{\rightarrow} 0 .
$$


Hence such a function $u_{j}$ verifies $\left.x u_{j}^{\prime}(x)\right|_{x=0}=0$, that is, its trace $x u_{j}^{\prime}$ vanishes at the origin. One can check that $J_{0}(2 \sqrt{\cdot}) \in H_{1 / 2}^{1}\left(\Omega_{j}\right)$. But on the contrary $Y_{0}(2 \sqrt{\cdot}) \notin H_{1 / 2}^{1}\left(\Omega_{j}\right)$, since the trace of $x Y_{0}^{\prime}(2 \sqrt{x})$ at the origin is equal to $\pi^{-1}$. To be more precise, on the scale of weighted Sobolev spaces $\left(H_{s}^{1}\left(\Omega_{j}\right)\right)_{s>0}$, one can check that the logarithmic function $\log |\cdot|$ belongs to $\bigcap_{\epsilon>0} H_{1 / 2+\epsilon}^{1}\left(\Omega_{j}\right)$ but not to $H_{1 / 2}^{1}\left(\Omega_{j}\right)$, which is the critical space on this scale. It follows that a generic solution $u$ does not belong to $H_{1 / 2}^{1}\left(\Omega_{j}\right)$, even if $u \in \bigcap_{\epsilon>0} H_{1 / 2+\epsilon}^{1}\left(\Omega_{j}\right)$.

We thus propose to represent $u$ on each subdomain $\Omega_{j}$ (i.e., on the propagative and non propagative regions separately) as the sum of a function $u_{j} \in H_{1 / 2}^{1}\left(\Omega_{j}\right)$ and of a complement term that is more singular. To characterize this singular term, we introduce the scalar $g$ at $x=0$ defined by $g=\left.x u^{\prime}(x)\right|_{x=0}$. We lift $g \in \mathbb{C}$ as a function $w_{g}$ with support in $\Omega$, in such a way that $w_{g}$ has a logarithmic derivative and verifies $\left.x w_{g}^{\prime}(x)\right|_{x=0}=g$. The solution $u$ to 1.4 on $\Omega$ then easily decomposes in regular and singular parts: one writes

$$
u(x)=u_{j}(x)+w_{g}(x) \quad x \in \Omega_{j}, \text { separately for } j=1,2 .
$$

In this Ansatz, the regular part is $u_{j} \in H_{1 / 2}^{1}\left(\Omega_{j}\right)$, and the singular part is $w_{g} \notin H_{1 / 2}^{1}\left(\Omega_{j}\right)$. This construction in $1 \mathrm{D}$ contains all the ideas behind the decomposition used in this work.

In $2 \mathrm{D}, \alpha(\mathbf{x})$ behaves like a signed distance to $\Sigma$. From the above 1D study, we expect a logarithmic growth in the normal direction to $\Sigma$. For a given bounded open set with Lipschitz boundary $\omega \subset \mathbb{R}^{2}$, we consider the weighted Sobolev space $H_{1 / 2}^{1}(\omega)$ containing the functions $v \in L^{2}(\omega)$ such that $\sqrt{\operatorname{dist}_{\partial \omega}} \nabla v \in L^{2}(\omega)^{2}$. With these notations, it will be possible to use Ansatz 1.5 in 2D in combination with the limit absorption principle $\nu=0^{+}$.

\subsection{Outline and main results}

To treat the singularity at $\Sigma$ which is the main objective of this work, we introduce some preliminary material. The first one is a specific decomposition of the unknown $u$ into a regular part and a singular part, as in 1.5. The second one is a characterization of the singular part following a limit absorption principle $\nu=0^{+}$. This is obtained in Section 4 and it is the most technical and original part of this work. The singular part $w_{g}^{+}$is characterized by the singular coefficient $g$, which is defined along $\Sigma$. The regular part is denoted as $\mathbf{u}=\left(u_{1}, u_{2}\right)$ and is defined by local problems on the subdomains $\Omega_{1}$ and $\Omega_{2}$ that involve the singular coefficient $g$. We introduce an auxiliary variable $h$ in the same space as $g$ and a Lagrange multiplier $\boldsymbol{\lambda}$ in the same space as $\mathbf{u}$. These functions are such that $(\mathbf{u}, g, h) \in V=Q \times H^{2}(\Sigma) \times H^{2}(\Sigma)$ and $\lambda \in Q=H_{1 / 2}^{1}\left(\Omega_{1}\right) \times H_{1 / 2}^{1}\left(\Omega_{2}\right)$. The validity of the decomposition of the solution relies on a technical lemma.

Lemma 1. Let $\omega \subset \mathbb{R}^{2}$ be a bounded open set with Lipschitz boundary. The weighted Sobolev space $H_{1 / 2}^{1}(\omega)$ is compactly embedded into $L^{2}(\omega)$.

This compact embedding is particular useful to show that a local problem used to define $u_{1}$ in the propagative sub-domain $\Omega_{1}$ is well posed. The local problem used to define $u_{2}$ in the non propagative sub-domain $\Omega_{2}$ is also well-posed, but the analysis can be simplified since the local problem is naturally coercive in $\Omega_{2}$. Using a family of explicit quasi-solutions for the regularized problem 1.3 , we implement the limit absorption principle in sections 3 and 4 . This allows us to formulate and prove the following result.

Theorem 2. The formal limit $\nu=0^{+}$of problem (1.3) admits a regularized mixed variational formulation

$$
\begin{aligned}
& \text { Find }(\mathbf{u}, g, h) \in V \text { and } \boldsymbol{\lambda} \in Q \text { such that } \\
& \left\{\begin{array}{llr}
a_{r}^{+}((\mathbf{u}, g, h),(\mathbf{v}, k, l))-\overline{b^{+}((\mathbf{v}, k, l), \boldsymbol{\lambda})} & =0, \quad \forall(\mathbf{v}, k, l) \in V, \\
b^{+}((\mathbf{u}, g, h), \boldsymbol{\mu}) & =\ell(\boldsymbol{\mu}), & \forall \boldsymbol{\mu} \in Q,
\end{array}\right.
\end{aligned}
$$

for $b^{+}$and $a_{r}^{+}$defined in (4.7) and 4.10 respectively. Moreover, this formulation is well-posed, in the sense that for all $f \in L^{2}(\overline{\Gamma)}$, there exists a unique solution $(\mathbf{u}, g, h) \in V$ and $\boldsymbol{\lambda} \in Q$, which depends continuously on $\|f\|_{L^{2}(\Gamma)}$.

The organization of this work is as follows. Next Section 2 is devoted to $2 \mathrm{D}$ geometrical notations and the functional setting. The variational characterization of the singularity with quasi-solutions and the $2 \mathrm{D}$ justification of the Ansatz (1.5) is provided in Section 3 . The mixed variational formulation (1.6) which combines all these ideas is defined and studied in Section 4 . Finally, in Section 5 , a discrete formulation 
(1.6) is constructed in the context of the classical finite element method. It leads to a new methods method for the numerical approximation of 1.1p. The numerical results illustrate on the one hand the robustness of the discretized formulation, and on the other hand the accuracy of the discrete solution.

\section{Geometry in dimension two}

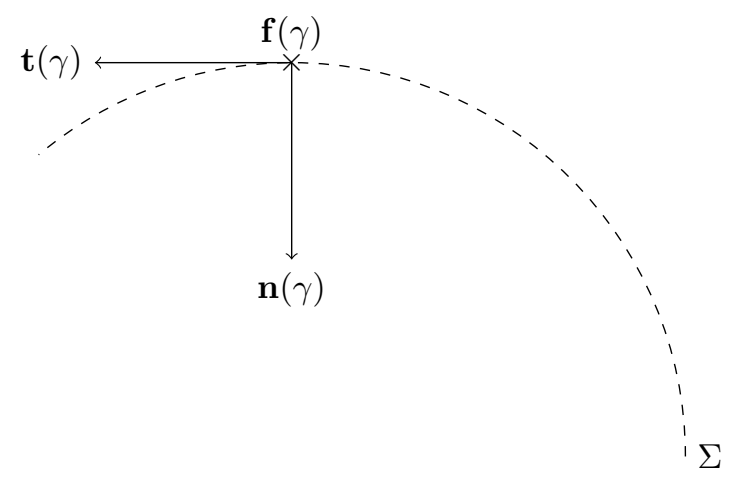

Figure 1: $\Sigma$ parametrization

We use a standard parametrization of the geometry, see [10].

\section{$2.1 \quad$ Notations}

The coefficient $\alpha$ is assumed to be smooth with enough derivatives, typically $\mathcal{C}^{2}$, and non degenerate in the sense that $\nabla \alpha(\mathbf{x}) \neq 0$ in the domain of interest. Under these conditions, we define the curve $\Sigma:=\left\{\mathbf{x} \in \mathbb{R}^{2}, \alpha(\mathbf{x})=0\right\}$, and to further simplify, we assume that $\Sigma$ is a closed simple line. We consider the parametrization $\mathbf{f}:[0,1] \rightarrow \mathbb{R}^{2}$ of $\Sigma$ illustrated in Fig. 2, with $\mathbf{f}(0)=\mathbf{f}(1)$ and $\mathbf{f}$ bijective between $[0,1)$ and $\Sigma$. We assume $\gamma$ is a curvilinear abscissa, that is $\left|\mathbf{f}^{\prime}(\gamma)\right|=1$. The curvature radius of $\Sigma$ at $\mathbf{f}(\gamma)$ is denoted $R(\gamma)$, and we note the minimal value of the curvature radius $R_{*}:=\min _{\gamma} R(\gamma)>0$. This quantity is well-defined for a continuous $R$. For a given $\gamma \in[0,1]$, the ingoing normal and tangent vectors to $\Sigma$ at $\mathbf{f}(\gamma)$ are denoted respectively $\mathbf{n}(\gamma)$ and $\mathbf{t}(\gamma)$. For all $\gamma \in[0,1]$ and $\sigma \in \mathbb{R}$, we set $\psi(\gamma, \sigma):=\mathbf{f}(\gamma)+\sigma \mathbf{n}(\gamma)$ which belongs to a neighbourhood of $\Sigma$ for small values of $\sigma$. It is known that $\psi$ is injective on $[0,1) \times\left(-R_{*}, R_{*}\right)$. We next define the tubular extension of $\Sigma$

$$
\Sigma_{\text {tub }}:=\psi\left([0,1) \times\left(-\frac{1}{2} R_{*}, \frac{1}{2} R_{*}\right)\right) \cap \Omega .
$$

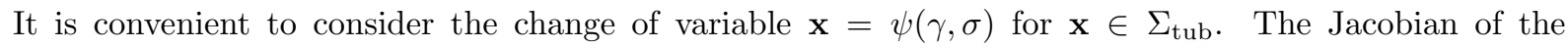
transformation

$$
p_{\Omega}(\gamma, \sigma):=\operatorname{det} \nabla \psi=\left|\mathbf{f}^{\prime}(\gamma)\right|-\frac{\sigma}{R(\gamma)}=1-\frac{\sigma}{R(\gamma)} \geq \frac{1}{2}
$$

is such that for $O \subset \Sigma_{\text {tub }}$

$$
\int_{O} v(\mathbf{x}) \mathrm{d} \mathbf{x}=\int_{\psi^{-1}(O)} v \circ \psi(\gamma, \sigma) p_{\Omega}(\gamma, \sigma) \mathrm{d} \sigma \mathrm{d} \gamma
$$

We will always use the notation $\nabla=\left(\partial_{x}, \partial_{y}\right)^{t}$, and one has

$$
\nabla \gamma(\mathbf{x})=\frac{\mathbf{t}(\gamma(\mathbf{x}))}{1-\sigma(\mathbf{x}) / R(\gamma(\mathbf{x}))} \quad \text { and } \quad \nabla \sigma(\mathbf{x})=\mathbf{n}(\gamma(\mathbf{x}))
$$

For any function $v$ we make the abuse of notation $v \circ \psi(\gamma, \sigma)=v(\gamma, \sigma)$. So we define $r(\gamma)=\partial_{\sigma} \alpha(\gamma, 0) \neq 0$, and we have the local expansion for small $\sigma$

$$
\alpha(\gamma, \sigma)=r(\gamma) \sigma+O\left(\sigma^{2}\right)
$$

with $r$ of constant sign. We suppose without loss of generality that $r<0$, therefore on a given $\Sigma_{\text {tub }}^{*} \subset \Sigma_{\text {tub }}$, one has $0<c_{*} \leq-\partial_{\sigma} \alpha \leq c^{*}$. In the sequel, we will consider the case $\Omega=\Sigma_{\text {tub. In particular, }}^{*}$.

$$
0<c_{*} \leq-\frac{\alpha(\gamma, \sigma)}{\sigma} \leq c^{*}, \quad \forall \psi(\gamma, \sigma) \in \Omega .
$$


We also define

$$
\Omega_{1}:=\psi\left([0,1) \times\left(-\frac{1}{2} R_{*}, 0\right)\right) \cap \Omega \quad \text { and } \quad \Omega_{2}:=\psi\left([0,1) \times\left(0, \frac{1}{2} R_{*}\right)\right) \cap \Omega,
$$

such that $\Omega=\Omega_{1} \cup \Sigma \cup \Omega_{2}$, and the exterior boundaries

$$
\Gamma_{1}:=\partial \Omega_{1} \cap \Gamma \quad \text { and } \quad \Gamma_{2}:=\partial \Omega_{2} \cap \Gamma
$$

where $\Gamma=\partial \Omega=\Gamma_{1} \cup \Gamma_{2}$. One has $\Omega_{1}=\{\alpha>0\}$ and $\Omega_{2}=\{\alpha<0\}$. Finally, we define the $L_{w}^{2}$ weighted norm on $\Sigma$ such that

$$
\|v\|_{L_{w}^{2}(\Sigma)}^{2}:=\int_{0}^{1}|v(\gamma, 0)|^{2} \frac{\mathbf{1}}{|r(\gamma)|} \mathrm{d} \gamma
$$

\subsection{Functional setting}

The unknowns are complex valued, and so are the considered functional spaces. We use the $\alpha$-weighted Sobolev spaces defined on $\Omega_{j}, j=1,2$ as

$$
H_{1 / 2}^{1}\left(\Omega_{j}\right):=\left\{u \in L^{2}\left(\Omega_{j}\right), \int_{\Omega_{j}}|\alpha(\mathbf{x})||\nabla u(\mathbf{x})|^{2} \mathrm{~d} \mathbf{x}<\infty\right\}
$$

endowed with the $\alpha$-weighted norm

$$
\|u\|_{H_{1 / 2}^{1}\left(\Omega_{j}\right)}:=\left(\|u\|_{L^{2}\left(\Omega_{j}\right)}^{2}+\|\sqrt{|\alpha|} \nabla u\|_{L^{2}\left(\Omega_{j}\right)}^{2}\right)^{1 / 2} .
$$

This norm is equivalent to the standard weighted $H_{1 / 2}^{1}$ norm involving the distance to a boundary 15 according to 2.4. The dual spaces, in the sense of the spaces of anti-linear maps into $\mathbb{C}$, are noted with a prime.

We recall the definition of T-coercivity as introduced in [6] which is an explicit realization of the inf-sup condition.

Definition 3. Let $H$ be a Hilbert space. Let $\mathrm{T}: H \rightarrow H$ be a continuous linear operator. A bilinear form $b$ defined on $H \times H$ is $\mathrm{T}$-coercive if there exists $C>0$ such that $|b(u, \mathrm{~T} u)| \geq C\|u\|_{H}^{2}$.

If $\mathrm{T}$ is a bijection, for all continuous linear forms $\ell$ defined on $H$, there exists a unique $u \in H$ such that $b(u, v)=\ell(v)$ for all $v \in H$. And as it is the case for coercive forms, up to a compact perturbation, $b$ is associated to a Fredholm operator of index 0. This last property implies that the associated variational formulation admits a unique solution if and only if uniqueness holds. This result will be used further. For any $x, y \in \mathbb{R}$, we note the complex logarithm $\log (x+i y):=\frac{1}{2} \log \left(x^{2}+y^{2}\right)-i \operatorname{atan}(x / y)$.

\section{Limit viscosity $\nu \rightarrow 0^{+}$solution}

\subsection{Variational formulations}

Because the sign of $\alpha$ changes on $\Sigma$, it is natural to separate the problem on each side of $\Sigma$, where it has a fixed sign. For these subproblems, we show a well-posedness result in the Hilbert space

$$
\begin{gathered}
Q:=H_{1 / 2}^{1}\left(\Omega_{1}\right) \times H_{1 / 2}^{1}\left(\Omega_{2}\right), \text { equipped with the norm } \\
\|\mathbf{u}\|_{Q}:=\left\|u_{1}\right\|_{H_{1 / 2}^{1}\left(\Omega_{1}\right)}+\left\|u_{2}\right\|_{H_{1 / 2}^{1}\left(\Omega_{2}\right)} \text { for } \mathbf{u}=\left(u_{1}, u_{2}\right) .
\end{gathered}
$$

Define the following problem

$$
\begin{aligned}
& \text { Find } \mathbf{u} \in Q \text { such that for all } \mathbf{v} \in Q \\
& b(\mathbf{u}, \mathbf{v})=\ell(\mathbf{v})
\end{aligned}
$$

where for $\mathbf{u}=\left(u_{1}, u_{2}\right)$ and $\mathbf{v}=\left(v_{1}, v_{2}\right) \in Q$,

$$
\begin{aligned}
b\left(\left(u_{1}, u_{2}\right),\left(v_{1}, v_{2}\right)\right) & :=\sum_{j=1,2}\left(\int_{\Omega_{j}}\left(\alpha \nabla u_{j} \cdot \overline{\nabla v_{j}}-u_{j} \overline{v_{j}}\right) \mathrm{d} \mathbf{x}+\int_{\Gamma_{j}} i \lambda u_{j} \overline{v_{j}} \mathrm{~d} s\right) \\
\ell\left(v_{1}, v_{2}\right) & :=\int_{\Gamma_{1}} f \overline{v_{1}} \mathrm{~d} s+\int_{\Gamma_{2}} f \overline{v_{2}} \mathrm{~d} s .
\end{aligned}
$$


Proposition 4. Let $\lambda>0$ and $f \in L^{2}(\Gamma)$. Problem 3.2 has a unique solution in $Q$.

Before proving Proposition 4, we prove Lemma 1 for $\omega=\Omega_{1}$.

Lemma 5. The weighted Sobolev space $H_{1 / 2}^{1}\left(\Omega_{1}\right)$ is compactly embedded into $L^{2}\left(\Omega_{1}\right)$.

Proof. Let $\left(u_{n}\right)_{n \in \mathbb{N}} \subset H_{1 / 2}^{1}\left(\Omega_{1}\right)$ be a bounded sequence. Up to a subsequence, $u_{n}$ weakly converges towards a limit in $H_{1 / 2}^{1}\left(\Omega_{1}\right)$, and substracting this limit to the sequence, one can consider $u_{n} \rightarrow 0$.

For all $\epsilon>0$, define $\Omega_{1}^{\epsilon}:=\left\{(\gamma, \sigma) \in \Omega_{1},|\sigma|<\epsilon\right\}$. The $H^{1}$ and $H_{1 / 2}^{1}$ norms are equivalent on any set $\Omega_{1} \backslash \Omega_{1}^{\epsilon}$ since the weight $\alpha$ is positively bounded below on this domain: so $\left\|u_{n}\right\|_{L^{2}\left(\Omega_{1} \backslash \Omega_{1}^{\epsilon}\right)} \rightarrow 0$.

To prove our claim, we show that as $\epsilon$ goes to 0 ,

$$
\int_{\Omega_{1}^{\epsilon}}\left|u_{n}\right|^{2} \mathrm{~d} \mathbf{x} \rightarrow 0 \quad \text { uniformly in } n \text {. }
$$

Introducing the value of $u_{n}$ on $\Gamma_{1}$, it yields

$$
\begin{aligned}
\int_{\Omega_{1}^{\epsilon}}\left|u_{n}\right|^{2} \mathrm{~d} \mathbf{x} & =\int_{\gamma} \int_{-\epsilon}^{0}\left|u_{n}(\gamma, \sigma)\right|^{2} p_{\Omega}(\gamma, \sigma) \mathrm{d} \sigma \mathrm{d} \gamma \\
& \leq 2 \int_{\gamma} \int_{-\epsilon}^{0}\left|u_{n}(\gamma, \sigma)-u_{n}(\gamma,-R)\right|^{2} p_{\Omega}(\gamma, \sigma) \mathrm{d} \sigma \mathrm{d} \gamma+2 \int_{\gamma} \int_{-\epsilon}^{0}\left|u_{n}(\gamma,-R)\right|^{2} p_{\Omega}(\gamma, \sigma) \mathrm{d} \sigma \mathrm{d} \gamma
\end{aligned}
$$

The second term is uniformly controlled by $\epsilon$ as

$$
\int_{\gamma} \int_{-\epsilon}^{0}\left|u_{n}(\gamma,-R)\right|^{2} p_{\Omega}(\gamma, \sigma) \mathrm{d} \sigma \mathrm{d} \gamma \leq C \epsilon\left\|u_{n}\right\|_{L^{2}\left(\Gamma_{1}\right)}^{2} \leq \tilde{C} \epsilon
$$

For the first term, a Cauchy-Schwarz argument applied twice gives

$$
\begin{aligned}
\int_{\gamma} \int_{-\epsilon}^{0}\left|u_{n}(\gamma, \sigma)-u_{n}(\gamma,-R)\right|^{2} p_{\Omega}(\gamma, \sigma) \mathrm{d} \sigma \mathrm{d} \gamma & =\int_{\gamma} \int_{-\epsilon}^{0}\left|\int_{-R}^{\sigma} \partial_{\sigma} u_{n}(\gamma, s) \mathrm{d} s\right|^{2} p_{\Omega}(\gamma, \sigma) \mathrm{d} \sigma \mathrm{d} \gamma \\
& \leq \int_{\gamma}^{0} \int_{-\epsilon}^{0}\left(\int_{-R}^{\sigma}|s|\left|\partial_{\sigma} u_{n}(\gamma, s)\right|^{2} \mathrm{~d} s\right) \log \left|\frac{R}{\sigma}\right| p_{\Omega}(\gamma, \sigma) \mathrm{d} \sigma \mathrm{d} \gamma \\
& \leq \int_{\gamma}^{0}\left(\int_{-R}^{0}|s|\left|\partial_{\sigma} u_{n}(\gamma, s)\right|^{2} \mathrm{~d} s\right)\left(\int_{-\epsilon}^{0} \log \left|\frac{R}{\sigma}\right| p_{\Omega}(\gamma, \sigma) \mathrm{d} \sigma\right) \mathrm{d} \gamma \\
& \leq \sup _{\gamma}^{0}\left(\int_{-\epsilon}^{0} \log \left|\frac{R}{\sigma}\right| p_{\Omega}(\gamma, \sigma) \mathrm{d} \sigma\right)\left(\int_{\gamma}^{0}|s|\left|\partial_{\sigma} u_{n}(\gamma, s)\right|^{2} \mathrm{~d} s \mathrm{~d} \gamma\right)
\end{aligned}
$$

The first term is such that

$$
\sup _{\gamma} \int_{-\epsilon}^{0} \log \left|\frac{R}{\sigma}\right| p_{\Omega}(\gamma, \sigma) \mathrm{d} \sigma \leq C \epsilon(1+|\log \epsilon|) \underset{\epsilon \rightarrow 0}{\rightarrow} 0,
$$

and the second one verifies

$$
\int_{\gamma} \int_{-R}^{0}|s|\left|\partial_{\sigma} u_{n}(\gamma, s)\right|^{2} \mathrm{~d} s \mathrm{~d} \gamma \leq C\left\|\alpha^{1 / 2} \nabla u_{n}\right\|_{L^{2}\left(\Omega_{1}\right)}^{2} \leq M
$$

with $M>0$ a constant independent of $n$. The result is established.

Proof. [Proposition 4] Let $\lambda>0$ and $f \in L^{2}(\Gamma)$.

First, $\ell$ is a continuous antilinear form on $Q$. In fact, for a given constant $C>0$,

$$
\begin{aligned}
\|\ell(\mathbf{v})\| & \leq\|f\|_{L^{2}(\Gamma)}\left(\left\|v_{1}\right\|_{L^{2}\left(\Gamma_{1}\right)}+\left\|v_{2}\right\|_{L^{2}\left(\Gamma_{2}\right)}\right) \\
& \leq C\|f\|_{L^{2}(\Gamma)}\|\mathbf{v}\|_{Q},
\end{aligned}
$$

since the $L^{2}$ norm on $\Gamma$ is controlled by the $H^{1}$ norm in a neighbourhood of $\Gamma$ in $\Omega$, and that $H^{1}$ and $H_{1 / 2}^{1}$ norms are equivalent away from $\Sigma$.

Second, the sesquilinear form $b$ is continuous on $Q \times Q$ since there exists a constant $C>0$ such that for all $\mathbf{u}, \mathbf{v} \in Q$,

$$
|b(\mathbf{u}, \mathbf{v})| \leq(2+\lambda C)\|\mathbf{u}\|_{Q}\|\mathbf{v}\|_{Q}
$$


Third, $b$ is the sum of coercive and compact forms. Denoting $b_{0}$ and $b_{1}$ the forms such that for $\mathbf{u}, \mathbf{v} \in Q$,

$$
b_{0}(\mathbf{u}, \mathbf{v}):=-2 \int_{\Omega_{1}} u_{1} \overline{v_{1}} \mathrm{~d} \mathbf{x} \quad \text { and } \quad b_{1}(\mathbf{u}, \mathbf{v}):=\left(u_{1}, v_{1}\right)_{H_{1 / 2}^{1}\left(\Omega_{1}\right)}-\left(u_{2}, v_{2}\right)_{H_{1 / 2}^{1}\left(\Omega_{2}\right)}+\sum_{j=1,2} \int_{\Gamma_{j}} i \lambda u_{j} \overline{v_{j}} \mathrm{~d} s
$$

one has the decomposition $b(\mathbf{u}, \mathbf{v})=b_{0}(\mathbf{u}, \mathbf{v})+b_{1}(\mathbf{u}, \mathbf{v})$. For all $\mathbf{v} \in Q$,

$$
\operatorname{Re} b_{1}(\mathbf{v}, \mathbf{T} \mathbf{v})=\|\mathbf{v}\|_{Q}^{2} \text { for } \quad \mathrm{T}=\left(\begin{array}{cc}
1 & 0 \\
0 & -1
\end{array}\right),
$$

hence $b_{1}$ is $\mathrm{T}$-coercive for the bijective operator $\mathrm{T}$.

The form $b_{0}$, equivalent to the $L^{2}$ scalar product on $\Omega_{1}$, is a compact perturbation of $b_{1}$ on $Q$ according to Lemma 5 for a bounded sequence $\left(\mathbf{v}^{n}\right)_{n \in \mathbb{N}} \subset Q$, and up to a subsequence, its first component $v_{1}^{n}$ converges in $L^{2}\left(\Omega_{1}\right)$.

Thus $b$ is associated to a Fredholm operator of zero index. The Fredholm alternative indicates it suffices to prove injectivity, in the sense that if $b(\mathbf{v},)=$.0 for a given $\mathbf{v} \in Q$, then $\mathbf{v}=0$, to have bijectivity.

Testing against $\left(0, v_{2}\right)$ and taking the real part, we obtain $\left\|v_{2}\right\|_{H_{1 / 2}^{1}\left(\Omega_{1}\right)}=0$. Testing against $\left(v_{1}, 0\right)$ we obtain first $\left\|v_{1}\right\|_{L^{2}\left(\Gamma_{1}\right)}=0$. For all $\epsilon>0$, the function $v_{1}$ also verifies the Helmholtz equation on $\Omega_{1} \backslash \Omega_{1}^{\epsilon}=\left\{\mathbf{x} \in \Omega_{1}, \operatorname{dist}_{\Sigma}(\mathbf{x}) \geq \epsilon\right\}$

$$
-\operatorname{div}(\alpha \nabla v)-v=0 .
$$

Going back to the variational formulation one finds that $\left.\partial_{n} v_{1}\right|_{\Gamma_{1}}=0$. The uniqueness continuation principle from partial Cauchy data implies that in $\Omega_{1} \backslash \Omega_{1}^{\epsilon}$ one has $v_{1}=0$. Letting $\epsilon$ go to 0 , the claim follows.

Since the weak formulation 3.2 of 1.1$]$ is restricted to $H_{1 / 2}^{1}$ solutions, it excludes $\log |\sigma|$ singularities as seen in Section 1.1. Thus, it will only allow us to describe the regular part. For the singular part, we will follow a limit absorption principle which relies on the regularized problem. The classical way is to introduce a complex shift $\alpha+i \nu$ [19, 5], and then pass to the limit $\nu \rightarrow 0^{+}$. We will prove that for $\nu=0^{+}$the limit solution decomposes into a regular part in the weighted space $Q$ plus a complementary singular part.

The remaining part of this section is devoted to show that the problem for $\nu>0$ is well-posed in $H^{1}(\Omega)$, which poses no real difficulties. For any $\nu>0$, problem 1.3 can be formulated in a variational way as

Find $u \in H^{1}(\Omega)$ such that for all $v \in H^{1}(\Omega)$,

$$
b^{\nu}(u, v)=\ell(v) \text {. }
$$

The sesquilinear form is

$$
b^{\nu}(u, v)=\int_{\Omega}((\alpha+i \nu) \nabla u \cdot \overline{\nabla v}-u \bar{v}) \mathrm{d} \mathbf{x}+\int_{\Gamma} i \lambda u \bar{v} \mathrm{~d} s
$$

and for purpose of simplicity we redefine another function $\ell$ that coincides with 3.3$)$ on $H^{1}(\Omega)$

$$
\ell(v):=\int_{\Gamma} f \bar{v} \mathrm{~d} s
$$

Proposition 6. Let $\nu>0, \lambda>0$ and $f \in L^{2}(\Gamma)$. The weak formulation (3.4) of problem (1.3) has a unique solution $u^{\nu}$ in $H^{1}(\Omega)$.

Proof. The continuity of forms $b^{\nu}$ and $\ell$ are straightforward, $\alpha$ being bounded and the $L^{2}$ norm on $\Gamma$ being controlled by the $H^{1}$ norm on $\Omega$. Let us show $b^{\nu}$ is coercive. For all $u \in H^{1}(\Omega)$,

$$
\operatorname{Im} b^{\nu}(u, u) \geq \nu\|\nabla u\|_{L^{2}(\Omega)^{2}}^{2} \quad \text { and } \quad \operatorname{Re} b^{\nu}(u, u) \geq-\|\alpha\|_{L^{\infty}(\Omega)} \int_{\Omega}|\nabla u|^{2} \mathrm{~d} \mathbf{x}+\|u\|_{L^{2}(\Omega)}^{2}
$$

so that for all $C>\|\alpha\|_{L^{\infty}(\Omega)} / \nu, \operatorname{Re}\left((1-i C) b^{\nu}(u, u)\right) \geq \min \left(1, C \nu-\|\alpha\|_{L^{\infty}(\Omega)}\right)\|u\|_{H^{1}(\Omega)}^{2}$ and $b^{\nu}$ is coercive. The Lax-Milgram Theorem can be applied to 3.4, which thus has a unique solution $u^{\nu}$ in $H^{1}(\Omega)$. 


\subsection{A family of quasisolutions}

For any $\nu>0$, we define a family of quasisolutions to problem 1.3 . It is composed of functions $w_{g}^{\nu}$ approximating the expected logarithmic singular behaviour as $\nu \rightarrow 0$ with data $g \in H^{2}(\Sigma)$ :

$$
w_{g}^{\nu}(\gamma, \sigma):=\frac{g(\gamma)}{r(\gamma)}\left(\frac{\log \left(r(\gamma)^{2} \sigma^{2}+\nu^{2}\right)}{2}-i \operatorname{atan}\left(\frac{r(\gamma) \sigma}{\nu}\right)\right)
$$

When applying the differential operators $(-\nabla \cdot((\alpha+i \nu) \nabla)-\mathrm{id})$ in $\Omega$ and $\left((\alpha+i \nu) \partial_{n}+i \lambda\right.$ id $)$ on $\Gamma$ to the family, we define the resulting quantities

$$
\left\{\begin{array}{lll}
q_{g}^{\nu}:=-\nabla \cdot\left((\alpha+i \nu) \nabla w_{g}^{\nu}\right)-w_{g}^{\nu} & \text { in } \Omega \\
z_{g}^{\nu}:=(\alpha+i \nu) \partial_{n} w_{g}^{\nu}+i \lambda w_{g}^{\nu} & \text { on } \Gamma .
\end{array}\right.
$$

Developing the equation in $\Omega$ from 3.7 and using $(2.2)$, one has

$$
\begin{aligned}
q_{g}^{\nu}(\gamma, \sigma)= & -\partial_{\gamma}\left((\alpha+i \nu) \partial_{\gamma} w_{g}^{\nu}\right)|\nabla \gamma|^{2}+(\alpha+i \nu) \partial_{\gamma} w_{g}^{\nu}\left(\partial_{\gamma} \nabla \gamma\right) \cdot \nabla \gamma \\
& +(\alpha+i \nu) \partial_{\sigma} w_{g}^{\nu}\left(\partial_{\gamma} \nabla \sigma\right) \cdot \nabla \gamma-\partial_{\sigma}\left((\alpha+i \nu) \partial_{\sigma} w_{g}^{\nu}\right)|\nabla \sigma|^{2}-w_{g}^{\nu} \\
= & {\left[\frac{-\partial_{\gamma}}{(1-\sigma / R(\gamma))^{2}}+\frac{\sigma R^{\prime}(\gamma) R(\gamma)}{(R(\gamma)-\sigma)^{3}}\right]\left((\alpha+i \nu)\left(\left(g^{\prime}(\gamma)-\frac{g(\gamma) r^{\prime}(\gamma)}{r(\gamma)}\right) w_{1}^{\nu}+\frac{g(\gamma)}{r(\gamma)} \frac{r^{\prime}(\gamma) \sigma}{r(\gamma) \sigma+i \nu}\right)\right) } \\
& +\left[-\partial_{\sigma}+\frac{1}{R(\gamma)-\sigma}\right]\left(g(\gamma) \frac{\alpha+i \nu}{r(\gamma) \sigma+i \nu}\right)-w_{g}^{\nu}(\gamma, \sigma)
\end{aligned}
$$

where $w_{1}^{\nu}(\gamma, \sigma)=r(\gamma)^{-1}\left(\frac{1}{2} \log \left(r(\gamma)^{2} \sigma^{2}+\nu^{2}\right)-i \operatorname{atan}\left(\frac{r(\gamma) \sigma}{\nu}\right)\right)$.

Proposition 7. Let $\nu \in(0,1)$ and $g \in H^{2}(\Sigma)$. The manufactured solution $w_{g}^{\nu}$, the right hand side $q_{g}^{\nu}$ belong to $L^{2}(\Omega)$ and the boundary term $z_{g}^{\nu}$ belongs to $L^{2}(\Gamma)$. Moreover, the bounds are uniform with respect to $\nu$.

Proof. Let $\nu \in(0,1)$ and $g \in H^{2}(\Sigma)$. The manufactured solution is defined in (3.7) as a product of $g$ which is an $H^{2}$ function with respect to $\gamma$, of $1 / r$ which is a bounded coefficient, and of the sum of a logarithm and of a bounded term. As a consequence, $w_{g}^{\nu}$ belongs to $L^{2}(\Omega)$, and the bound is uniform with respect to $\nu$.

Assumption 2.3 ensures that the fractions

$$
\frac{\alpha+i \nu}{r(\gamma) \sigma+i \nu}, \quad \frac{\sigma}{r(\gamma) \sigma+i \nu}, \quad \frac{\sigma(\alpha+i \nu)}{(r(\gamma) \sigma+i \nu)^{2}}
$$

are bounded, i.e. $O(1)$, for small $\sigma$ with a constant independent of $\nu$. For the term

$$
\partial_{\sigma}\left[\frac{\alpha+i \nu}{r(\gamma) \sigma+i \nu}\right]=\frac{\partial_{\sigma} \alpha}{r(\gamma) \sigma+i \nu}-\frac{r(\gamma)(\alpha+i \nu)}{(r(\gamma) \sigma+i \nu)^{2}}
$$

one finds once again that

$$
\left|\frac{\partial_{\sigma} \alpha}{r(\gamma) \sigma+i \nu}-\frac{r(\gamma)(\alpha+i \nu)}{(r(\gamma) \sigma+i \nu)^{2}}\right| \leq \frac{\left|r(\gamma) \sigma \partial_{\sigma} \alpha-r(\gamma) \alpha\right|}{(r(\gamma) \sigma)^{2}}+\frac{\nu\left|\partial_{\sigma} \alpha-r(\gamma)\right|}{2 \nu|r(\gamma) \sigma|}=O(1) \quad \text { for small } \sigma .
$$

Refering to 3.9$), q_{g}^{\nu}$ thus amounts to a sum of square integrable terms independently of the value of $\nu$. Finally, for $z_{g}^{\nu}$, since it involves the function $w_{g}^{\nu}$ and its derivatives away from the curve $\Sigma$, it is indeed in $L^{2}(\Gamma)$ with a bound that is uniform with respect to $\nu$.

Lemma 8. Let $g \in H^{2}(\Sigma)$. As $\nu \rightarrow 0^{+}$, the $L^{2}$ limit of the manufactured functions defined above are

$$
\left\{\begin{aligned}
w_{g}^{+}(\gamma, \sigma)= & \frac{g(\gamma)}{r(\gamma)}\left(\log |r(\gamma) \sigma|-\frac{i \pi}{2} \operatorname{sign}(r(\gamma) \sigma)\right) \quad \text { in } L^{2}(\Omega), \\
q_{g}^{+}(\gamma, \sigma)= & {\left[\frac{-\partial_{\gamma}}{(1-\sigma / R(\gamma))^{2}}+\frac{\sigma R^{\prime}(\gamma) R(\gamma)}{(R(\gamma)-\sigma)^{3}}\right] } \\
& \left(\left(\frac{g^{\prime}(\gamma)}{r(\gamma)}-\frac{g(\gamma) r^{\prime}(\gamma)}{r^{2}(\gamma)}\right) \alpha\left(\log |r(\gamma) \sigma|-\frac{i \pi}{2} \operatorname{sign}(r(\gamma) \sigma)\right)+\frac{g(\gamma) r^{\prime}(\gamma)}{r(\gamma)} \sigma \frac{\alpha}{r(\gamma) \sigma}\right) \\
& +\left[-\partial_{\sigma}+\frac{1}{R(\gamma)-\sigma}\right]\left(g(\gamma) \frac{\alpha}{r(\gamma) \sigma}\right)-w_{g}^{+}(\gamma, \sigma) \text { in } L^{2}(\Omega), \\
z_{g}^{+}(\gamma, \sigma)= & \alpha \partial_{n} w_{g}^{+}+i \lambda w_{g}^{+} \text {in } L^{2}(\Gamma) .
\end{aligned}\right.
$$


Proposition 9. For $g \in H^{2}(\Sigma)$, the associated manufactured solutions depend in the following way on $g$ $\left\|w_{g}^{+}\right\|_{L^{2}(\Omega)} \leq C\|g\|_{L^{2}(\Sigma)},\left\|\alpha \nabla w_{g}^{+}\right\|_{L^{2}(\Omega)^{2}} \leq C\|g\|_{H^{1}(\Sigma)},\left\|q_{g}^{+}\right\|_{L^{2}(\Omega)} \leq C\|g\|_{H^{2}(\Sigma)}$, and $\left\|z_{g}^{+}\right\|_{L^{2}(\Gamma)} \leq C\left\|_{g}\right\|_{H^{1}(\Sigma)}$, for four positive constants $C$ independent of $g$.

Remark 10. For any non trivial $g \in H^{2}(\Sigma)$, function $w_{g}^{+}$does not belong to $H_{1 / 2}^{1}(\Omega)$. On the other hand, for any smooth function $\phi$ that vanishes on $\Sigma$, the term $\left(|\alpha|^{1 / 2} \nabla w_{g}^{+}\right) \phi$ is square integrable. In fact, the most singular term in $\int_{\Omega}|\alpha|\left|\nabla w_{g}^{+}\right|^{2} \phi^{2}$ is

$$
\int_{0}^{1} \int_{-R}^{R}|\alpha| \frac{|g(\gamma)|^{2}}{r^{2}(\gamma) \sigma^{2}} \phi^{2}(\gamma, \sigma) p_{\Omega}(\gamma, \sigma) \mathrm{d} \sigma \mathrm{d} \gamma
$$

which is indeed integrable since $\alpha(\gamma, \sigma)=r(\gamma) \sigma+O\left(\sigma^{2}\right)$. Therefore $w_{g}^{+} \in \bigcap_{\epsilon>0} H_{1 / 2+\epsilon}^{1}(\Omega)$.

Proposition 11. Let $g \in H^{2}(\Sigma)$. The $\alpha$-weighted flux of $w_{g}^{+}$on $\Sigma$ is equal to $g$, in the sense that for all $h \in L^{2}(\Sigma)$,

$$
\int_{\gamma} \alpha(\gamma, 0) \nabla w_{g}^{+}(\gamma, 0) \cdot \mathbf{n}(\gamma) \overline{h(\gamma)} \mathrm{d} \gamma=\int_{\gamma} g(\gamma) \overline{h(\gamma)} \mathrm{d} \gamma
$$

Proof. Let $\nu>0$. For all $g \in H^{2}(\Sigma)$, and $\gamma \in[0,1]$,

$$
(\alpha(\gamma, 0)+i \nu) \nabla w_{g}^{\nu}(\gamma, 0) \cdot \mathbf{n}(\gamma)=\frac{\alpha(\gamma, 0)+i \nu}{r(\gamma) \times 0+i \nu} g(\gamma)|\mathbf{n}(\gamma)|^{2}=g(\gamma)
$$

According to Proposition $9 .(\alpha+i \nu) \nabla w_{g}^{\nu}$ converges weakly in $L^{2}(\Sigma)$ towards $\alpha \nabla w_{g}^{+}$, so the result is proven.

Remark 12. This last proposition is essential for the decomposition in regular and singular parts. We will introduce a singular coefficient $g$ supported on $\Sigma$ representing the $\alpha$-weighted flux over the curve $\Sigma$ of the whole solution of (1.1). Going back to the $1 D$ case, it corresponds to the fact $x Y_{0}^{\prime}$ has a trace at $x=0$ and in this case the unknown $g$ is reduced to the coefficient $b$, see subSection 1.1 .

\subsection{Decomposition of the solution in regular and singular parts}

According to the Ansatz introduced in Section 1.1. we decompose $u$ as

$$
u=\mid \begin{array}{ll}
u_{1}-w_{g}^{+}, & \text {in } \Omega_{1}, \\
u_{2}-w_{g}^{+}, & \text {in } \Omega_{2},
\end{array}
$$

with the singular coefficient $g \in H^{2}(\Sigma)$ yet to be characterized, and for $j=1,2$, the regular part $u_{j} \in H_{1 / 2}^{1}\left(\Omega_{j}\right)$ such that

$$
\left\{\begin{aligned}
-\nabla \cdot\left(\alpha \nabla u_{j}\right)-u_{j} & =q_{g}^{+}, & & \text {in } \Omega_{j} \\
\alpha \partial_{n} u_{j}+i \lambda u_{j} & =f+z_{g}^{+}, & & \text {on } \Gamma_{j} \\
\alpha \partial_{n} u_{j} & =0, & & \text { on } \Sigma .
\end{aligned}\right.
$$

For a fixed $g$, these equations rewrite in a classical variational way

$$
\begin{aligned}
& \text { Find } \mathbf{u} \in Q \text { such that for all } \mathbf{v} \in Q \\
& b(\mathbf{u}, \mathbf{v})=\ell_{g}(\mathbf{v}),
\end{aligned}
$$

where this time the antilinear form $\ell_{g}$ is defined by

$$
\ell_{g}\left(v_{1}, v_{2}\right):=\sum_{j=1,2} \int_{\Omega_{j}} q_{g}^{+} \overline{v_{j}} \mathrm{~d} \mathbf{x}+\int_{\Gamma_{j}}\left(f+z_{g}^{+}\right) \overline{v_{j}} \mathrm{~d} s .
$$

For $f=0$ and $g \in H^{2}(\Sigma)$, we denote the solution $\mathbf{u}(g)=\left(u_{1}(g), u_{2}(g)\right)$.

Proposition 13. Let $\lambda>0, f \in L^{2}(\Gamma)$ and $g \in H^{2}(\Sigma)$. There exists a unique solution $\left(u_{1}, u_{2}\right) \in Q$ solution of the weak formulation (3.13) of (3.12). The solution is such that

$$
\left\|u_{1}\right\|_{H_{1 / 2}^{1}\left(\Omega_{1}\right)} \leq C\left(\|g\|_{H^{2}(\Sigma)}+\|f\|_{L^{2}(\Gamma)}\right) \quad \text { and } \quad\left\|u_{2}\right\|_{H_{1 / 2}^{1}\left(\Omega_{2}\right)} \leq C\left(\|g\|_{H^{2}(\Sigma)}+\|f\|_{L^{2}(\Gamma)}\right),
$$

for constants $C>0$ that are independent of $f$ and $g$. 
Proof. Under the assumption that $\ell_{g}$ is continuous, the proof of Prop. 4 shows that the problem is wellposed for any $\lambda>0, f \in L^{2}(\Gamma)$ and $g \in H^{2}(\Sigma)$. And the continuity of $\ell_{g}$ is immediate considering Prop. 9.

Testing against $\left(u_{1}, 0\right)$ and $\left(0, u_{2}\right)$ respectively, we obtain the bounds

$$
\left\|u_{1}\right\|_{H_{1 / 2}^{1}\left(\Omega_{1}\right)}-\left\|u_{1}\right\|_{L^{2}\left(\Omega_{1}\right)} \leq C\left(\|g\|_{H^{2}(\Sigma)}+\|f\|_{L^{2}(\Gamma)}\right),
$$

and

$$
\left\|u_{2}\right\|_{H_{1 / 2}^{1}\left(\Omega_{2}\right)} \leq C\left(\|g\|_{H^{2}(\Sigma)}+\|f\|_{L^{2}(\Gamma)}\right) .
$$

Let us now precise the bound (3.16). We show that there exists a constant $C>0$ that is independent of $f$ and $g$ such that

$$
\left\|u_{1}\right\|_{L^{2}\left(\Omega_{1}\right)} \leq C\left(\|f\|_{L^{2}\left(\Gamma_{1}\right)}+\|g\|_{H^{2}(\Sigma)}\right) .
$$

We proceed by contradiction as in e.g. [11. Consider there exists sequences $\left(f_{k}\right)_{k \in \mathbb{N}} \subset L^{2}(\Gamma),\left(g_{k}\right)_{k \in \mathbb{N}} \subset$ $H^{2}(\Sigma)$ and $\left(u_{1, k}\right)_{k \in \mathbb{N}} \subset H_{1 / 2}^{1}\left(\Omega_{1}\right)$ that verify for all $k \in \mathbb{N}$,

$$
\left\{\begin{aligned}
-\nabla \cdot\left(\alpha \nabla u_{1, k}\right)-u_{1, k} & =q_{g_{k}}^{+}, & & \text {in } \Omega_{1} \\
\alpha \partial_{n} u_{1, k}+i \lambda u_{1, k} & =f_{k}+z_{g_{k}}^{+}, & & \text {on } \Gamma_{1} \\
\alpha \partial_{n} u_{1, k} & =0, & & \text { on } \Sigma
\end{aligned}\right.
$$

and such that $\left\|u_{1, k}\right\|_{L^{2}\left(\Omega_{1}\right)}=1$ for all $k$ and $\left\|f_{k}\right\|_{L^{2}(\Gamma)}+\left\|g_{k}\right\|_{H^{2}(\Sigma)} \rightarrow 0$. Using relation (3.16), we get that $\left(u_{1, k}\right)_{k \in \mathbb{N}}$ is bounded in $H_{1 / 2}^{1}\left(\Omega_{1}\right)$ norm. Therefore there exists $u_{1}^{*} \in H_{1 / 2}^{1}\left(\Omega_{1}\right)$ towards which, up to a subsequence, $u_{1, k}$ converges weakly in $H_{1 / 2}^{1}\left(\Omega_{1}\right)$ and strongly in $L^{2}\left(\Omega_{1}\right)$ according to Lemma 5 . Thus $u_{1}^{*}$ is the weak solution of

$$
\left\{\begin{aligned}
-\nabla \cdot\left(\alpha \nabla u_{1}\right)-u_{1} & =0, & \text { in } \Omega_{1} \\
\alpha \partial_{n} u_{1}+i \lambda u_{1} & =0, & \text { on } \Gamma_{1} \\
\alpha \partial_{n} u_{1} & =0, & \text { on } \Sigma
\end{aligned}\right.
$$

which means $u_{1}^{*}=0$. But this contradicts the fact that $\left\|u_{1}^{*}\right\|_{L^{2}\left(\Omega_{1}\right)}=1$.

So the bound (3.16) can be expressed exclusively in terms of the $H_{1 / 2}^{1}\left(\Omega_{1}\right)$ norm of $u_{1}$ as

$$
\left\|u_{1}\right\|_{H_{1 / 2}^{1}\left(\Omega_{1}\right)} \leq C\left(\|g\|_{H^{2}(\Sigma)}+\|f\|_{L^{2}(\Gamma)}\right)
$$

and the proof is ended.

It is now necessary to find how to characterize the transmission condition on $\Sigma$, quantified by the unknown $g$, to close the system.

\section{A mixed variational formulation of the limit problem}

\subsection{Energy estimates}

Let $u^{\nu}$ denote the solution of 1.3 . We introduce the set of compactly supported, smooth and positive cutoff around $\Sigma$ functions depending only on $\sigma$, and not on $\gamma$

$$
\mathcal{C}_{0,+}^{1}(\Omega)=\left\{\varphi \in \mathcal{C}_{0}^{1}(\Omega), \partial_{\gamma} \varphi=0, \varphi \geq 0,\left.\varphi\right|_{\Sigma}=1\right\} .
$$

Let $\varphi \in \mathcal{C}_{0,+}^{1}(\Omega)$. We introduce a new unknown $h \in H^{2}(\Sigma)$, which corresponds to a dual variable associated to the unknown $g$. For a given $h$, testing the equation verified by $u^{\nu}+w_{h}^{\nu}$ against $\overline{\left(u^{\nu}+w_{h}^{\nu}\right)} \varphi$ gives

$$
\int_{\Omega}\left(-\nabla \cdot\left((\alpha+i \nu) \nabla\left(u^{\nu}+w_{h}^{\nu}\right)\right) \overline{\left(u^{\nu}+w_{h}^{\nu}\right)} \varphi-\left|u^{\nu}+w_{h}^{\nu}\right|^{2} \varphi\right) \mathrm{d} \mathbf{x}=\int_{\Omega} q_{h}^{\nu} \overline{\left(u^{\nu}+w_{h}^{\nu}\right)} \varphi \mathrm{d} \mathbf{x} .
$$

Integrating by parts, it yields

$\int_{\Omega}\left((\alpha+i \nu)\left|\nabla\left(u^{\nu}+w_{h}^{\nu}\right)\right|^{2} \varphi+(\alpha+i \nu) \overline{\left(u^{\nu}+w_{h}^{\nu}\right)} \nabla\left(u^{\nu}+w_{h}^{\nu}\right) \cdot \nabla \varphi-\left|u^{\nu}+w_{h}^{\nu}\right|^{2} \varphi\right) \mathrm{d} \mathbf{x}=\int_{\Omega} q_{h}^{\nu} \overline{\left(u^{\nu}+w_{h}^{\nu}\right)} \varphi \mathrm{d} \mathbf{x}$,

wich implies

$$
\operatorname{Im} \int_{\Omega}\left((\alpha+i \nu)\left(u^{\nu}+w_{h}^{\nu}\right) \nabla \overline{\left(u^{\nu}+w_{h}^{\nu}\right)} \cdot \nabla \varphi+q_{h}^{\nu} \overline{\left(u^{\nu}+w_{h}^{\nu}\right)} \varphi\right) \mathrm{d} \mathbf{x}=\int_{\Omega} \nu\left|\nabla\left(u^{\nu}+w_{h}^{\nu}\right)\right|^{2} \mathrm{~d} \mathbf{x} \geq 0 .
$$


Definition 14. Let $\nu>0, \varphi \in \mathcal{C}_{0,+}^{1}(\Omega)$. For all $u \in H^{1}(\Omega)$ and $h \in H^{2}(\Sigma)$, define the quadratic form

$$
\mathcal{J}^{\nu}(u, h)=\operatorname{Im} \int_{\Omega}\left((\alpha+i \nu)\left(u+w_{h}^{\nu}\right) \nabla \overline{\left(u+w_{h}^{\nu}\right)} \cdot \nabla \varphi+q_{h}^{\nu} \overline{\left(u+w_{h}^{\nu}\right)} \varphi\right) \mathrm{d} \mathbf{x} .
$$

We also define the Hilbert space

$$
\begin{gathered}
V:=Q \times H^{2}(\Sigma) \times H^{2}(\Sigma), \text { equipped with the norm } \\
\|(\mathbf{u}, g, h)\|_{V}:=\|\mathbf{u}\|_{Q}+\|g\|_{H^{2}(\Sigma)}+\|h\|_{H^{2}(\Sigma)} .
\end{gathered}
$$

and the limit quadratic form such that for all $(\mathbf{u}, g, h) \in V$ with $\mathbf{u}=\left(u_{1}, u_{2}\right)$

$$
\mathcal{J}^{+}(\mathbf{u}, g, h)=\sum_{j=1,2} \operatorname{Im} \int_{\Omega_{j}}\left(\alpha\left(u_{j}-w_{g-h}^{+}\right) \nabla \overline{\left(u_{j}-w_{g-h}^{+}\right)} \cdot \nabla \varphi+q_{h}^{+} \overline{\left(u_{j}-w_{g-h}^{+}\right)} \varphi\right) \mathrm{d} \mathbf{x} .
$$

For a given $(\mathbf{u}, g, h)$, the quantity $\mathcal{J}^{+}(\mathbf{u}, g, h)$ is the formal pointwise limit of $\mathcal{J}^{\nu}(u, h)$ where $\left.u\right|_{\Omega_{j}}$ is a regularization of $u_{j}-w_{g}^{+}$for $j=1$, 2. Since relation 4.2 holds, the idea is now to minimize $\mathcal{J}^{+}$under the constraint that $(\mathbf{u}, g)$ verifies equation 3.13. We define for this purpose the following Lagrangian on $V \times Q$, such that for all $(\mathbf{u}, g, h) \in V$ and $\mathbf{v} \in Q$

$$
\mathcal{L}^{+}(\mathbf{u}, g, h, \mathbf{v})=\mathcal{J}^{+}(\mathbf{u}, g, h)+\operatorname{Im}\left(b(\mathbf{u}, \mathbf{v})-\ell_{g}(\mathbf{v})\right) .
$$

\subsection{Mixed variational formulation}

To begin with, define $b^{+}$the sesquilinear form on $V \times Q$ such that for all $(\mathbf{u}, g, h) \in V$ and $\mathbf{v} \in Q$,

$$
b^{+}((\mathbf{u}, g, h), \mathbf{v})=\sum_{j=1,2}\left(\int_{\Omega_{j}}\left(\alpha \nabla u_{j} \cdot \nabla \overline{v_{j}}-\left(u_{j}+q_{g}^{+}\right) \overline{v_{j}}\right) \mathrm{d} \mathbf{x}+\int_{\Gamma_{j}}\left(i \lambda u_{j}-z_{g}^{+}\right) \overline{v_{j}} \mathrm{~d} s\right) .
$$

Remark 15. On $V \times Q$, it holds that $b^{+}((\mathbf{u}, g, h), \mathbf{v})=b^{+}((\mathbf{u}, g, 0), \mathbf{v})=b(\mathbf{u}, \mathbf{v})-\ell_{g}(\mathbf{v})+\ell(\mathbf{v})$.

Define $a^{+}$a sesquilinear form on $V \times V$ that verifies $\operatorname{Im} a^{+}=d \mathcal{J}^{+}$. We choose the form defined for all $(\mathbf{u}, g, h),(\mathbf{v}, k, l) \in V$ by

$$
\begin{aligned}
a^{+}((\mathbf{u}, g, h),(\mathbf{v}, k, l))= & \sum_{j=1,2} \int_{\Omega_{j}}\left(\alpha\left(u_{j}-w_{g-h}^{+}\right) \nabla \overline{\left(v_{j}-w_{k-l}^{+}\right)} \cdot \nabla \varphi+q_{h}^{+} \overline{\left(v_{j}-w_{k-l}^{+}\right)} \varphi\right) \mathrm{d} \mathbf{x} \\
& -\int_{\Omega_{j}}\left(\alpha \overline{\left(v_{j}-w_{k-l}^{+}\right)} \nabla\left(u_{j}-w_{g-h}^{+}\right) \cdot \nabla \varphi+\overline{q_{l}^{+}}\left(u_{j}-w_{g-h}^{+}\right) \varphi\right) \mathrm{d} \mathbf{x} .
\end{aligned}
$$

Note that $a^{+}$is anti-hermitian.

The Euler-Lagrange equations associated to the minimization of 4.6 have the following structure

$$
\begin{aligned}
& \text { Find }(\mathbf{u}, g, h) \in V \text { and } \boldsymbol{\lambda} \in Q \text { such that } \\
& \left\{\begin{array}{llr}
a^{+}((\mathbf{u}, g, h),(\mathbf{v}, k, l))-\overline{b^{+}((\mathbf{v}, k, l), \boldsymbol{\lambda})} & =0, \quad \forall(\mathbf{v}, k, l) \in V, \\
b^{+}((\mathbf{u}, g, h), \boldsymbol{\mu}) & =\ell(\boldsymbol{\mu}), & \forall \boldsymbol{\mu} \in Q .
\end{array}\right.
\end{aligned}
$$

We will see that with an arbitrary small regularization in $g$ and $h$ for the form $a^{+}$, this form is T-coercive on $V$, which allows us to apply the classical results of [3] and lead to the conclusion that the regularized problem is well-posed.

For $\rho, \mu \in \mathbb{R}^{+}$, we introduce the regularized form on $V \times V$

$$
a_{r}^{+}((\mathbf{u}, g, h),(\mathbf{v}, k, l))=a^{+}((\mathbf{u}, g, h),(\mathbf{v}, k, l))+i\left(-\rho(g, k)_{H^{2}(\Sigma)}+\mu\left(h^{\prime \prime}, l^{\prime \prime}\right)_{L^{2}(\Sigma)}\right) .
$$

Theorem 16. Let $\lambda>0, f \in L^{2}(\Gamma)$, and $\rho, \mu>0$. The regularized formulation of 4.9

$$
\begin{aligned}
& \text { Find }(\mathbf{u}, g, h) \in V \text { and } \boldsymbol{\lambda} \in Q \text { such that } \\
& \left\{\begin{array}{llrl}
a_{r}^{+}((\mathbf{u}, g, h),(\mathbf{v}, k, l))-\overline{b^{+}((\mathbf{v}, k, l), \boldsymbol{\lambda})} & =0, & \forall(\mathbf{v}, k, l) \in V, \\
b^{+}((\mathbf{u}, g, h), \boldsymbol{\mu}) & =\ell(\boldsymbol{\mu}), & \forall \boldsymbol{\mu} \in Q,
\end{array}\right.
\end{aligned}
$$

admits a unique solution.

Remark 17. Theorem 16 means that the regularization in $\nu$ across the curve $\Sigma$ has been replaced by a regularization in $\rho$ and $\mu$ along $\Sigma$. 


\subsection{Proof of the well-posedness}

Denote $B^{+}: V \rightarrow Q^{\prime}$ the linear continuous operator such that for all $(\mathbf{v}, k, l) \in V$ and $\boldsymbol{\mu} \in Q$,

$$
\left(B^{+}(\mathbf{v}, k, l), \boldsymbol{\mu}\right)_{Q^{\prime}, Q}=b^{+}((\mathbf{v}, k, l), \boldsymbol{\mu}),
$$

and let $K:=\operatorname{ker} B^{+}$. Denote $A_{K K^{\prime}}^{+}: K \rightarrow K^{\prime}$ the linear continuous operator such that for all $(\mathbf{u}, g, h),(\mathbf{v}, k, l) \in K$,

$$
\left(A_{K K^{\prime}}^{+}(\mathbf{u}, g, h),(\mathbf{v}, k, l)\right)_{K^{\prime}, K}=a_{r}^{+}((\mathbf{u}, g, h),(\mathbf{v}, k, l)),
$$

The proof of Theorem 16 will consist in applying the following classical result.

Theorem 18 (Theorem 4.2.2 of Boffi-Brezzi-Fortin $[3]$ in $\mathbb{C}$ ). For any $\kappa \in V^{\prime}$ and $\varkappa \in Q^{\prime}$, the mixed system

$$
\begin{aligned}
& \text { Find }(\mathbf{u}, g, h) \in V \text { and } \boldsymbol{\lambda} \in Q \text { such that } \\
& \qquad \begin{array}{llr}
a_{r}^{+}((\mathbf{u}, g, h),(\mathbf{v}, k, l))-\overline{b^{+}((\mathbf{v}, k, l), \boldsymbol{\lambda})} & =(\kappa,(\mathbf{v}, k, l))_{V^{\prime}, V}, & \forall(\mathbf{v}, k, l) \in V, \\
b^{+}((\mathbf{u}, g, h), \boldsymbol{\mu}) & =(\varkappa, \boldsymbol{\mu})_{Q^{\prime}, Q}, & \forall \boldsymbol{\mu} \in Q .
\end{array}
\end{aligned}
$$

has a unique solution if and only if $A_{K K^{\prime}}^{+}$is an isomorphism from $K$ to $K^{\prime}$ and if $\operatorname{Im} B^{+}=Q^{\prime}$.

Proposition 19. Operator $B^{+}$is onto $Q^{\prime}$.

Proof. This is a consequence of Prop. 13. Indeed, according to (4.7), $B^{+}$is such that

$$
\left(B^{+}(\mathbf{u}, g, h), \boldsymbol{\mu}\right)_{Q^{\prime}, Q}=b(\mathbf{u}, \boldsymbol{\mu})-\sum_{j=1,2}\left(\int_{\Omega_{j}} q_{g}^{+} \overline{\mu_{j}} \mathrm{~d} \mathbf{x}+\int_{\Gamma_{j}} z_{g}^{+} \overline{\mu_{j}} \mathrm{~d} s\right) .
$$

For any $\varkappa \in Q^{\prime}$, it has been proven that for all $g \in H^{2}(\Sigma)$, there exists $\mathbf{u} \in Q$ such that

$$
b(\mathbf{u}, \boldsymbol{\mu})=\sum_{j=1,2}\left(\int_{\Omega_{j}} q_{g}^{+} \overline{\mu_{j}} \mathrm{~d} \mathbf{x}+\int_{\Gamma_{j}} z_{g}^{+} \overline{\mu_{j}} \mathrm{~d} s\right)+(\varkappa, \boldsymbol{\mu})_{Q^{\prime}, Q} \quad \forall \boldsymbol{\mu} \in Q .
$$

As a result, operator $B^{+}$is onto $Q^{\prime}$.

Proposition 20. The kernel $K$ of operator $B^{+}$can be described as

$$
K=\left\{(\mathbf{u}, g, h) \in V, \quad \mathbf{u}(g)=\left(u_{1}(g), u_{2}(g)\right)\right\} .
$$

Proof. As the last component in $H^{2}(\Sigma)$ is a silent variable for $B^{+}$, it is not constrained. Necessarily $(\mathbf{u}, g)$ is such that $b(\mathbf{u}, \boldsymbol{\mu})=\ell_{g}(\boldsymbol{\mu})$ for all $\boldsymbol{\mu} \in Q$, where the boundary term $f \in L^{2}(\Gamma)$ is taken equal to 0. Refering to Prop. 13, this is verified for all $g \in H^{2}(\Sigma)$ by $\mathbf{u}(g)=\left(u_{1}(g), u_{2}(g)\right)$.

Let us now address the properties verified by $a^{+}$.

Proposition 21. For all $(\mathbf{u}, g, h),(\mathbf{v}, k, l) \in K$,

$$
\begin{aligned}
a^{+}((\mathbf{u}, g, h),(\mathbf{v}, k, l))= & \sum_{j=1,2} \int_{\Omega_{j}} q_{h}^{+} \overline{\left(v_{j}-w_{k-l}^{+}\right)}-\overline{q_{l}^{+}}\left(u_{j}-w_{g-h}^{+}\right) \mathrm{d} \mathbf{x} \\
& +\int_{\Gamma_{j}} z_{h}^{+} \overline{\left(v_{j}-w_{k-l}^{+}\right)}-\overline{z_{l}^{+}}\left(u_{j}-w_{g-h}^{+}\right)-2 i \lambda\left(u_{j}-w_{g-h}^{+} \overline{\left(v_{j}-w_{k-l}^{+}\right)} \mathrm{d} s\right.
\end{aligned}
$$

and $a^{+}$is independent of the cutoff $\varphi$, as long as $\varphi \in \mathcal{C}_{0,+}^{1}(\Omega)$, see 4.1. .

Proof. Let $(\mathbf{u}, g, h),(\mathbf{v}, k, l) \in K$, with $\mathbf{u}=\left(u_{1}, u_{2}\right)=\left(u_{1}(g), u_{2}(g)\right)$ and $\mathbf{v}=\left(v_{1}, v_{2}\right)=\left(u_{1}(k), u_{2}(k)\right)$ according to Prop. 20. Remark that

$$
\begin{aligned}
a^{+}((\mathbf{u}, g, h),(\mathbf{v}, k, l))= & \sum_{j=1,2} \int_{\Omega_{j}}\left(\alpha\left(u_{j}-w_{g-h}^{+}\right) \nabla \overline{\left(v_{j}-w_{k-l}^{+}\right)} \cdot \nabla(\varphi-1)+q_{h}^{+} \overline{\left(v_{j}-w_{k-l}^{+}\right)}(\varphi-1)\right) \mathrm{d} \mathbf{x} \\
& -\int_{\Omega_{j}}\left(\alpha \overline{\left(v_{j}-w_{k-l}^{+}\right)} \nabla\left(u_{j}-w_{g-h}^{+}\right) \cdot \nabla(\varphi-1)+\overline{q_{l}^{+}}\left(u_{j}-w_{g-h}^{+}\right)(\varphi-1)\right) \mathrm{d} \mathbf{x} \\
& +\int_{\Omega_{j}}\left(q_{h}^{+} \overline{\left(v_{j}-w_{k-l}^{+}\right)}-\overline{q_{l}^{+}}\left(u_{j}-w_{g-h}^{+}\right)\right) \mathrm{d} \mathbf{x} .
\end{aligned}
$$


Since $\left.\varphi\right|_{\Sigma}=1$ and $\alpha\left|\nabla w_{\cdot}^{+}\right|^{2}=\frac{c_{0}(\gamma, \sigma)}{\sigma}+c_{1}(\gamma, \sigma)$ with $c_{0}, c_{1} \in L^{2}(\Omega)$, the terms in $\alpha\left|\nabla w_{.}^{+}\right|^{2}(\varphi-1)$ are integrable thanks to Hardy's inequality, which ensures that $\frac{\varphi-1}{\sigma}$ is square integrable on $\Omega$. Therefore the quantity

$$
\sum_{j=1,2} \int_{\Omega_{j}} \alpha \nabla \overline{\left(v_{j}-w_{k-l}^{+}\right)} \cdot \nabla\left(u_{j}-w_{g-h}^{+}\right)(\varphi-1) \mathrm{d} \mathbf{x}
$$

is well defined. The first two lines above thus rewrite

$$
\begin{aligned}
A:= & \sum_{j=1,2} \int_{\Omega_{j}}\left(\alpha\left(u_{j}-w_{g-h}^{+}\right) \nabla \overline{\left(v_{j}-w_{k-l}^{+}\right)} \cdot \nabla(\varphi-1)+q_{h}^{+} \overline{\left(v_{j}-w_{k-l}^{+}\right)}(\varphi-1)\right) \mathrm{d} \mathbf{x} \\
& -\int_{\Omega_{j}}\left(\alpha \overline{\left(v_{j}-w_{k-l}^{+}\right)} \nabla\left(u_{j}-w_{g-h}^{+}\right) \cdot \nabla(\varphi-1)+\overline{q_{l}^{+}}\left(u_{j}-w_{g-h}^{+}\right)(\varphi-1)\right) \mathrm{d} \mathbf{x} \\
= & \sum_{j=1,2} \int_{\Omega_{j}}\left(\alpha \nabla \overline{\left(v_{j}-w_{k-l}^{+}\right)} \cdot \nabla\left(\left(u_{j}-w_{g-h}^{+}\right)(\varphi-1)\right)+q_{h}^{+} \overline{\left(v_{j}-w_{k-l}^{+}\right)}(\varphi-1)\right) \mathrm{d} \mathbf{x} \\
& \left.-\int_{\Omega_{j}}\left(\alpha \nabla\left(u_{j}-w_{g-h}^{+}\right) \cdot \nabla \overline{\left(v_{j}-w_{k-l}^{+}\right)}(\varphi-1)\right)+\overline{q_{l}^{+}}\left(u_{j}-w_{g-h}^{+}\right)(\varphi-1)\right) \mathrm{d} \mathbf{x} \\
= & \sum_{j=1,2} \int_{\Omega_{j}}\left(\alpha \nabla \overline{\left(v_{j}-w_{k-l}^{+}\right)} \cdot \nabla\left(\left(u_{j}-w_{g-h}^{+}\right)(\varphi-1)\right)+\left(u_{j}-w_{g-h}^{+}+q_{h}^{+}\right) \overline{\left(v_{j}-w_{k-l}^{+}\right)}(\varphi-1)\right) \mathrm{d} \mathbf{x} \\
& -\int_{\Omega_{j}}\left(\alpha \nabla\left(u_{j}-w_{g-h}^{+}\right) \cdot \nabla\left(\overline{\left(v_{j}-w_{k-l}^{+}\right)}(\varphi-1)\right)+\overline{\left(v_{j}-w_{k-l}^{+}+q_{l}^{+}\right)}\left(u_{j}-w_{g-h}^{+}\right)(\varphi-1)\right) \mathrm{d} \mathbf{x} .
\end{aligned}
$$

Since on each $\Omega_{j} u_{j}-w_{g-h}^{+}$verifies weakly $-\nabla \cdot\left(\alpha \nabla\left(u_{j}-w_{g-h}^{+}\right)\right)-\left(u_{j}-w_{g-h}^{+}\right)=q_{h}^{+}$with boundary conditions on $\Gamma_{j} \alpha \partial_{n}\left(u_{j}-w_{g-h}^{+}\right)+i \lambda\left(u_{j}-w_{g-h}^{+}\right)=f+z_{h}^{+}$, and since $v_{j}-w_{k-l}^{+}$verifies the corresponding relations for $g=k, h=l$ and $u_{j}=v_{j}=u_{j}(k)$, integrating by parts and using that $\left.(\varphi-1)\right|_{\Sigma}=0$ it yields

$$
\begin{aligned}
A & =\sum_{j=1,2} \int_{\Gamma_{j}}\left(\overline{\left(v_{j}-w_{k-l}^{+}\right)} \alpha \partial_{n}\left(u_{j}-w_{g-h}^{+}\right)-\left(u_{j}-w_{g-h}^{+}\right) \alpha \partial_{n} \overline{\left(v_{j}-w_{k-l}^{+}\right)}\right) \mathrm{d} s \\
& =\sum_{j=1,2} \int_{\Gamma_{j}}\left(\overline{\left(v_{j}-w_{k-l}^{+}\right)}\left(-i \lambda\left(u_{j}-w_{g-h}^{+}\right)+z_{h}^{+}\right)-\left(u_{j}-w_{g-h}^{+}\right) \overline{\left(-i \lambda\left(v_{j}-w_{k-l}^{+}\right)+z_{l}^{+}\right)}\right) \mathrm{d} s
\end{aligned}
$$

and the result is proven.

Lemma 22. For all $g \in H^{2}(\Sigma)$,

$$
\operatorname{Im} \int_{\Omega} q_{g}^{+} \overline{w_{g}^{+}} \mathrm{d} \mathbf{x}=\pi\|g\|_{L_{w}^{2}(\Sigma)}^{2}-\operatorname{Im} \int_{\Gamma}\left(z_{g}^{+}-i \lambda w_{g}^{+}\right) \overline{w_{g}^{+}} \mathrm{d} s .
$$

Proof. For all $\epsilon>0$, define $\psi_{\epsilon}$ a cutoff of $\Sigma$, a real valued piecewise affine function of $\sigma$ such that $\psi_{\epsilon}(\gamma, \sigma)=0$ if $|\sigma|<\epsilon / 2$ and $\psi_{\epsilon}(\gamma, \sigma)=1$ if $|\sigma|>\epsilon$. For all $g \in H^{2}(\Sigma)$, using $\psi_{\epsilon}$ and integrating by parts,

$$
\begin{aligned}
\operatorname{Im} \int_{\Omega} q_{g}^{+} \overline{w_{g}^{+}} \mathrm{d} \mathbf{x} & =\lim _{\epsilon \rightarrow 0} \operatorname{Im} \int_{\Omega} q_{g}^{+} \overline{w_{g}^{+}} \psi_{\epsilon} \mathrm{d} \mathbf{x} \\
& =\lim _{\epsilon \rightarrow 0} \operatorname{Im}\left(\int_{\Omega}\left(\alpha\left|\nabla w_{g}^{+}\right|^{2} \psi_{\epsilon}+\alpha \overline{w_{g}^{+}} \nabla w_{g}^{+} \cdot \nabla \psi_{\epsilon}-\left|w_{g}^{+}\right|^{2} \psi_{\epsilon}\right) \mathrm{d} \mathbf{x}-\int_{\Gamma} \alpha \partial_{n} w_{g}^{+} \overline{w_{g}^{+}} \mathrm{d} s\right) \\
& =\lim _{\epsilon \rightarrow 0} \operatorname{Im}\left(\int_{\Omega} \alpha \overline{w_{g}^{+}} \nabla w_{g}^{+} \cdot \nabla \psi_{\epsilon} \mathrm{d} \mathbf{x}-\int_{\Gamma}\left(z_{g}^{+}-i \lambda w_{g}^{+}\right) \overline{w_{g}^{+}} \mathrm{d} s\right) .
\end{aligned}
$$

Let us now compute this first integral on $\Omega$ for a given $\epsilon>0$. Expressing the functions in terms of $(\gamma, \sigma)$,

$$
\begin{aligned}
& \operatorname{Im} \int_{\Omega} \alpha \overline{w_{g}^{+}} \nabla w_{g}^{+} \cdot \nabla \psi_{\epsilon} \mathrm{dx}= \operatorname{Im} \int_{\gamma} \int_{-\epsilon}^{-\epsilon / 2} \frac{-2|g(\gamma)|^{2}}{\epsilon r(\gamma)} \frac{\alpha}{r(\gamma) \sigma}\left(\log |r(\gamma) \sigma|-i \frac{\pi}{2} \operatorname{sign}(r(\gamma))\right) p_{\Omega}(\gamma, \sigma) \mathrm{d} \sigma \mathrm{d} \gamma \\
&+\operatorname{Im} \int_{\gamma} \int_{\epsilon}^{\epsilon} \frac{2|g(\gamma)|^{2}}{\epsilon r(\gamma)} \frac{\alpha}{r(\gamma) \sigma}\left(\log |r(\gamma) \sigma|+i \frac{\pi}{2} \operatorname{sign}(r(\gamma))\right) p_{\Omega}(\gamma, \sigma) \mathrm{d} \sigma \mathrm{d} \gamma \\
&= \operatorname{Im} \int_{\gamma} \int_{\epsilon / 2}^{\epsilon}\left[\frac{-2|g(\gamma)|^{2}}{\epsilon r(\gamma)} \frac{\alpha}{r(\gamma) \sigma}\left(\log |r(\gamma) \sigma|-i \frac{\pi}{2} \operatorname{sign}(r(\gamma))\right) p_{\Omega}(\gamma,-\sigma)\right. \\
&\left.+\frac{2|g(\gamma)|^{2}}{\epsilon r(\gamma)} \frac{\alpha}{r(\gamma) \sigma}\left(\log |r(\gamma) \sigma|+i \frac{\pi}{2} \operatorname{sign}(r(\gamma))\right) p_{\Omega}(\gamma, \sigma)\right] \mathrm{d} \sigma \mathrm{d} \gamma
\end{aligned}
$$


for the weight $p_{\Omega}(\gamma, \sigma)=1-\sigma / R(\gamma)$ defined in 2.1). Identifying the imaginary part, it follows

$$
\begin{aligned}
\operatorname{Im} \int_{\Omega} \alpha \overline{w_{g}^{+}} \nabla w_{g}^{+} \cdot \nabla \psi_{\epsilon} \mathrm{d} \mathbf{x} & =\int_{\gamma} \int_{\epsilon / 2}^{\epsilon} \frac{2|g(\gamma)|^{2}}{\epsilon|r(\gamma)|} \frac{\alpha}{r(\gamma) \sigma} \pi \mathrm{d} \sigma \mathrm{d} \gamma \\
& \rightarrow \underset{\epsilon \rightarrow 0}{ } \int_{\gamma} \frac{|g(\gamma)|^{2}}{|r(\gamma)|} \pi \mathrm{d} \gamma
\end{aligned}
$$

which is equal to $\pi\|g\|_{L_{w}^{2}(\Sigma)}$ The proof is ended.

With this technical lemma we can now state the following.

Proposition 23. For all $(\mathbf{u}(g), g, h) \in K$,

$a^{+}((0,0, h),(0,0, h))=2 i \pi\|h\|_{L_{w}^{2}(\Sigma)}^{2} \quad$ and $\quad a^{+}((\mathbf{u}(g), g, 0),(\mathbf{u}(g), g, 0))=-2 i \lambda \sum_{j=1,2} \int_{\Gamma_{j}}\left|u_{j}(g)-w_{g}^{+}\right|^{2} \mathrm{~d} s$.

Proof. Let $h \in H^{2}(\Sigma)$. According to 4.15,

$$
\begin{aligned}
a^{+}((0,0, h),(0,0, h)) & =\int_{\Omega}\left(q_{h}^{+} \overline{w_{h}^{+}}-\overline{q_{h}^{+}} w_{h}^{+}\right) \mathrm{d} \mathbf{x}+\int_{\Gamma}\left(z_{h}^{+} \overline{w_{h}^{+}}-\overline{z_{h}^{+}} w_{h}^{+}-2 i \lambda\left|w_{h}^{+}\right|^{2}\right) \mathrm{d} s \\
& =2 i \operatorname{Im}\left(\int_{\Omega} q_{h}^{+} \overline{w_{h}^{+}} \mathrm{d} \mathbf{x}+\int_{\Gamma}\left(z_{h}^{+} \overline{w_{h}^{+}}-i \lambda\left|w_{h}^{+}\right|^{2}\right) \mathrm{d} s\right) .
\end{aligned}
$$

By Lemma 22 it is thus equal to $2 i \pi\|h\|_{L_{w}^{2}(\Sigma)}^{2}$. Now let $g \in H^{2}(\Sigma)$ and $\mathbf{u}=\mathbf{u}(g) \in Q$. Relying again on 4.15 , it follows

$$
a^{+}((\mathbf{u}(g), g, 0),(\mathbf{u}(g), g, 0))=\sum_{j=1,2} \int_{\Gamma_{j}}-2 i \lambda\left|u_{j}-w_{g}^{+}\right|^{2} \mathrm{~d} s
$$

Proposition 24. Let $\rho, \mu>0$. The sesquilinear form $a_{r}^{+}$defined in 4.10 is $\mathrm{T}$-coercive on $K$ in the sense that there exists a positive constant $C$ such that for all $(\mathbf{u}(g), g, h) \in K$,

$$
\operatorname{Im} a_{r}^{+}((\mathbf{u}(g), g, h), \mathrm{T}(\mathbf{u}(g), g, h)) \geq C\|(\mathbf{u}(g), g, h)\|_{V}
$$

for $\mathrm{T}:(\mathbf{u}, g, h) \in V \mapsto(-\mathbf{u},-g, h) \in V$. In particular, the operator $A_{K K^{\prime}}^{+}$defined in 4.12 is an isomorphism.

Proof. Let $\rho, \mu>0$, and $(\mathbf{u}(g), g, h) \in K$. Using the definition of form $a_{r}^{+}$and the fact that $a^{+}$is anti-hermitian, it follows from Proposition 23 that

$$
\begin{aligned}
\operatorname{Im} a_{r}^{+}((\mathbf{u}(g), g, h),(-\mathbf{u}(g),-g, h))= & 2 \pi\|h\|_{L_{w}^{2}(\Sigma)}^{2}+2 \lambda \sum_{j=1,2}\left\|u_{j}(g)-w_{g}^{+}\right\|_{L^{2}\left(\Gamma_{j}\right)}^{2} \\
& +\rho\|g\|_{H^{2}(\Sigma)}^{2}+\mu|h|_{H^{2}(\Sigma)}^{2} \\
\geq & C\left(\|g\|_{H^{2}(\Sigma)}^{2}+\|h\|_{H^{2}(\Sigma)}^{2}\right)
\end{aligned}
$$

for a $C>0$. Using Proposition 13 on the control of $\|\mathbf{u}(g)\|_{Q}$ by $\|g\|_{H^{2}(\Sigma)}$, we are able to conclude.

Proof. [Theorem 16] The hypotheses of Theorem 18 have been verified in Propositions 19 and 24.

Remark 25. An equivalence between $g \mapsto \lambda\left\|u_{j}(g)-w_{g}^{+}\right\|_{L^{2}(\Gamma)}$ and the $L^{2}(\Sigma)$ norm can be obtained on the kernel of $a^{+}$, see [18].

\section{Numerical illustration}

The general objectives of this section are to confirm the theoretical analysis by showing numerical results for the approximation of singular solutions of system 4.11 and to show that the mixed variational method developed in this work is compatible with standard finite element solvers, such as Freefem $++[14]$ in our case. To do so, we construct simple reference analytical solutions with and without a logarithmic singularity and use them for numerical error measurements. 


\subsection{Construction of analytical solutions}

We construct an analytical solution on a simplified model. Dropping out the 0 order term, which is a compact perturbation, in (1.1), one has

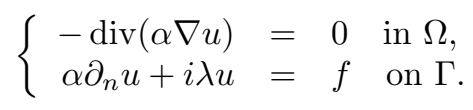

Let $\Omega$ be $(-1,1) \times(-1,1)$ with periodic boundary conditions at $y= \pm 1$, with $\Sigma=\{x=0\}$ and $\alpha=x$. A Fourier decomposition in the $y$-direction $u(\mathbf{x})=\sum_{k \in \mathbb{Z}} u_{k}(x) \exp (i k \pi y)$ yields for all modes

$$
x u_{k}^{\prime \prime}+u_{k}^{\prime}-x(k \pi)^{2} u_{k}=0 \text { in }(-1,1) .
$$

The general solution is

$$
u_{k}(x)=\mid \begin{array}{lr}
a_{k} I_{0}(k \pi x)+b_{k} K_{0}(k \pi x) & \forall k \neq 0, \\
a_{0}+b_{0}\left(\log |x|-\frac{i \pi}{2} \operatorname{sign}(x)\right) & k=0,
\end{array}
$$

where the modified Bessel functions are given by

$$
\left\{\begin{aligned}
I_{0}(x) & =J_{0}(i x), \\
K_{0}(x) & =-\frac{\pi}{2}\left(i J_{0}(i x)+Y_{0}(-i x)\right),
\end{aligned}\right.
$$

see Section 1.1 for the Bessel functions. We consider four test cases which respective solutions are

$$
u^{1}(\mathbf{x})=1, \quad u^{2}(\mathbf{x})=\log |x|-\frac{i \pi}{2} \operatorname{sign}(x), \quad u^{3}(\mathbf{x})=e^{i \pi y} I_{0}(\pi x) \quad \text { and } \quad u^{4}(\mathbf{x})=e^{i \pi y} K_{0}(\pi x) .
$$

The functions $u^{1}$ and $u^{2}$ are solutions for the $k=0$ mode. For these two functions, one computes easily the associated $(\mathbf{u}, g, h) \in V$ and $\boldsymbol{\lambda} \in Q$ solution of the variational formulation 4.11). According to the decomposition (3.11), one has

$$
\left\{\begin{array}{lll}
u_{j}^{1}=1 & \text { and } & g^{1}=0 \\
u_{j}^{2}=0 & \text { and } & g^{2}=-1
\end{array}\right.
$$

and in both cases, $h=g$ and $\lambda_{j}=u_{j} \varphi$, where $\varphi$ is the cut-off function that localizes near $\Sigma$. In the illustration below, the cut-off function is $\varphi(x)=\exp \left(4 x^{2} /\left(4 x^{2}-1\right)\right) \mathbf{1}_{(-1 / 2,1 / 2)}(x)$.

On the other hand, the functions $u^{3}$ and $u^{4}$ are solutions for the $k=1$ mode. One can compute the corresponding $(\mathbf{u}, g, h) \in V$ and $\boldsymbol{\lambda} \in Q$ as well.

The functions $u^{1}$ and $u^{3}$ are regular, while the functions $u^{2}$ and $u^{4}$ exhibit the logarithmic singularity which is the object of this study.

With $\lambda=1$, the boundary condition source term $f$ in 5.1 is determined accordingly and

$f^{1}(\mathbf{x})=i, f^{2}(\mathbf{x})=\left(1+\frac{\pi}{2}\right) \operatorname{sign}(x), f^{3}(\mathbf{x})=e^{i \pi y}\left(\pi I_{0}^{\prime}(\pi x)+i I_{0}(\pi x)\right), f^{4}(\mathbf{x})=e^{i \pi y}\left(\pi K_{0}^{\prime}(\pi x)+i K_{0}(\pi x)\right)$.

\subsection{Principle of the discretization}

The tests were implemented using the Freefem $++[14$ code. Freefem ++ offers a large choice of bidimensional finite elements, but does not allow so far to discretize a generic bilinear form like $a_{h}\left(u_{h}, v_{h}\right)$ where $u_{h}$ belongs to a 1D FE space and $v_{h}$ to a $2 \mathrm{D} \mathrm{FE}$ space. Since we need this feature, we have decided to focus on simple geometry and to use a penalization method to constrain a 2D FE space to unidimensionality. For this reason, the $H^{2}(\Sigma)$ space is discretized using 2D P3 Hsieh-Clough-Tocher (HCT) elements [8] penalized in the $x$-direction on a $2 \mathrm{D}$ triangular mesh denoted $\Sigma^{M}$. The upper script $M$ stands for the number of triangles that lie on $\Sigma$. The more standard $H^{1}\left(\Omega_{j}\right) 2 \mathrm{D}$ spaces are discretized using P1 elements on uniform triangular meshes of $\Omega_{j}$ denoted $\Omega_{j}^{N}$, where the upper script $N$ stands for the number of edges on each $\Gamma_{j}$. In the presented test cases, the parameters are $M=4$ for the P3 elements and $N=40$ for the P1 elements.

The discretization of 4.11] leads to the linear system

$$
\mathrm{AU}=\mathrm{L}
$$


with $\mathrm{U}=\left(\underline{u}_{1}, \underline{u}_{2}, \underline{g}, \underline{h}, \underline{\lambda}_{1}, \underline{\lambda}_{2}\right)$ the coefficients of the solution in the appropriate $\mathrm{FE}$ bases, and where $\mathrm{A}$ and $\mathrm{L}$ have block matricial structures

$$
\mathrm{A}=\left(\begin{array}{cccccc}
A_{1} & 0 & A_{g 1} & A_{h 1} & B_{1} & 0 \\
* & A_{2} & A_{g 2} & A_{h 2} & 0 & B_{2} \\
* & * & A_{g} & A_{h k} & B_{1 k} & B_{2 k} \\
* & * & * & A_{h} & 0 & 0 \\
* & * & * & * & 0 & 0 \\
* & * & * & * & * & 0
\end{array}\right), \quad \mathrm{L}=\left(\begin{array}{c}
0 \\
0 \\
0 \\
0 \\
\underline{\ell}_{1} \\
\underline{\ell}_{2}
\end{array}\right)
$$

As a consequence of the structure (4.11) and because the sesquilinear form $a^{+} 4.8$ is anti-hermitian, the matrix $A$ is anti-hermitian $A=-\bar{A}^{t}$. Note that the penalization used to achieve unidimensionality is performed in a similar way to 4.10, so that the anti-hermitian nature of the matrix is preserved.

\subsection{Numerical results}

The numerical solution is obtained by solving the linear system (5.4)-(5.5). With the numerical implementation described above, we observe that the matrices are non-singular and the computations run smoothly.

\subsubsection{Numerical errors}

In Table 1 we present the relative errors in $L^{2}(\Omega)$ norms for the four test cases on the total solution $u$ of (5.1). We observe a relative error of order $10^{-2}$ for all problems even for this coarse mesh. We also observe that the error magnitude is slightly smaller for the case 1 in mode 0 and case 3 in mode 1 . Our interpretation is that it is due to the regularity of $u^{1}$ and $u^{3}$, whereas $u^{2}$ and $u^{4}$ have logarithmic singularities.

\begin{tabular}{c|cccc}
\multicolumn{1}{l|}{} & case 1 & case 2 & case 3 & case 4 \\
\hline$\frac{\left\|u_{e x}-u_{\text {num }}\right\|_{L^{2}}}{\left\|u_{e x}\right\|_{L^{2}}}$ & 0.017 & 0.047 & 0.008 & 0.024
\end{tabular}

Table 1: For the four test cases of (5.2), relative $L^{2}(\Omega)$ error between the exact solution $u_{e x}$ and its approximation $u_{\text {num }}=u_{j}-w_{g}^{+}$.

In Table 2, we present the block residual errors of AU $-\mathrm{L}$. The block residuals are defined from (5.5) as the residuals for each of the 6 unknowns. It allows a more accurate description of the residual error. We perform the test for the test cases 1 and 2, which means that $\mathrm{U}$ takes the two exact values

$$
\mathrm{U}^{1}=\left(\begin{array}{c}
1_{N}^{\Omega_{1}} \\
1_{N}^{\Omega_{2}} \\
0 \\
0 \\
\varphi_{N}^{\Omega_{1}} \\
\varphi_{N}^{\Omega_{2}}
\end{array}\right) \quad \text { and } \quad \mathrm{U}^{2}=\left(\begin{array}{c}
0 \\
0 \\
-1_{M}^{\Sigma} \\
-1_{M}^{\Sigma} \\
0 \\
0
\end{array}\right)
$$

In this expression cf. (5.3), $1_{N}^{\Omega_{j}}$ and $\varphi_{N}^{\Omega_{j}}$ are the coefficients of the P1 interpolations of the functions 1 and $\varphi$ in $H^{1}\left(\Omega_{j}\right)$, and $1_{M}^{2}$ are the coefficients of 1 in the HCT space.

A priori, a residual error is the result of three main contributions which are an interpolation error, a penalization error, and errors due to the approximation of the bilinear forms.

We observe in Table 2 that all block residual errors are close to machine precision, except for the first four blocks in test case 1. After inspection of the structure of A and the nature of the exact solutions (5.6), our interpretation is that when machine precision is reached, the only significant error comes from interpolation errors.

\subsubsection{Plot of the numerical solutions in cases 3 and 4}

The imaginary part of the numerical approximation of the solutions $u^{3}$ and $u^{4}$ is shown on the right part of figures 2 and 3 The exact solutions, which are Bessel functions modulated in the direction $y$, are shown on the left part of the figures. The trace of the 2D FE mesh is also visible, together with 


\begin{tabular}{rccc} 
& norm & case 1 & case 2 \\
\hline$u_{1}$ block & $L^{2}(\Omega)$ & 0.0419775 & $7.76013 \mathrm{e}-16$ \\
\hline$u_{2}$ block & $L^{2}(\Omega)$ & 0.0491617 & $7.14962 \mathrm{e}-16$ \\
\hline$g$ block & $L^{2}(\Sigma)$ & 0.0360422 & $3.86971 \mathrm{e}-12$ \\
\hline$h$ block & $L^{2}(\Sigma)$ & 0.0361913 & $4.37914 \mathrm{e}-12$ \\
\hline$\lambda_{1}$ block & $L^{2}(\Omega)$ & $7.04875 \mathrm{e}-15$ & $7.68808 \mathrm{e}-16$ \\
\hline$\lambda_{2}$ block & $L^{2}(\Omega)$ & $7.29193 \mathrm{e}-15$ & $2.70813 \mathrm{e}-15$
\end{tabular}

Table 2: Residual errors in $L^{2}$ norms for the two first test cases.

the vertical line $\Sigma$. In Fig. 2, we observe that the numerical solution on the right is qualitatively and quantitatively very similar to the exact one on the left. The Fig. 3 is of greater interest since the exact solution presents the logarithmic singular behaviour. Qualitatively, the results are very similar and the logarithmic singularity seems to be correctly captured by the numerical solution. Quantitatively, the $L^{2}$ norm of the relative error is small, as reported in Table 1, even if a small discrepancy is visible, partly due to a different scaling between both representations.
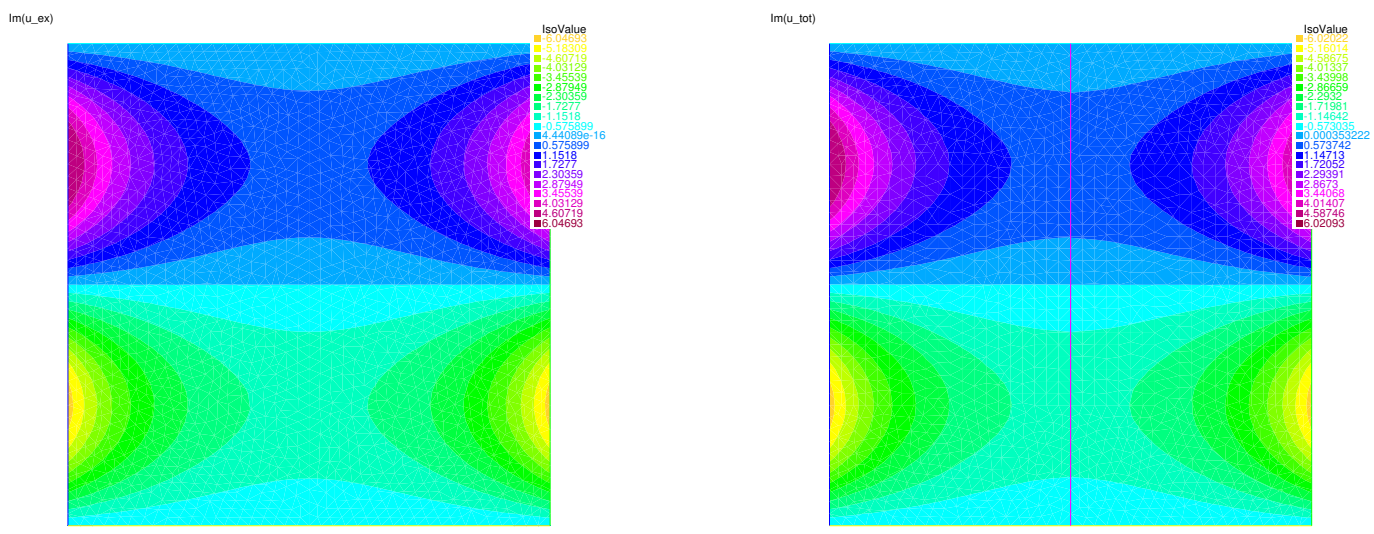

Figure 2: Imaginary parts of the exact solution $u^{3}(\mathbf{x})=e^{i \pi y} I_{0}(\pi x)$ (left) and its approximation (right).
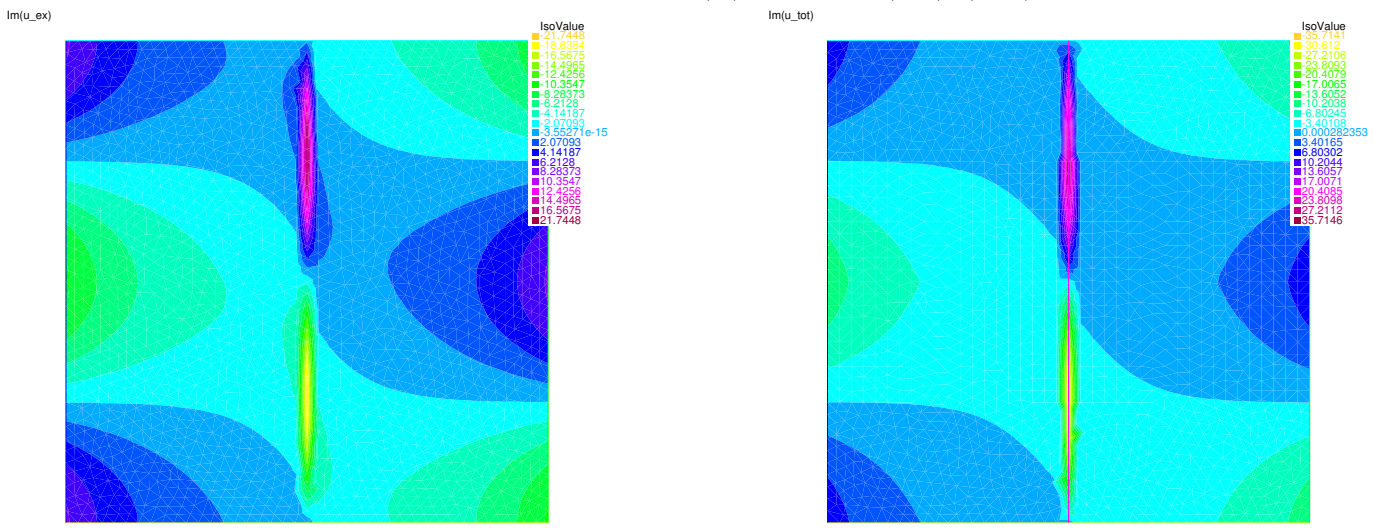

Figure 3: Imaginary parts of the exact solution $u^{4}(\mathbf{x})=e^{i \pi y} K_{0}(\pi x)$ (left) and its approximation (right).

\subsubsection{Plot of the numerical solution of the full problem}

We now consider the initial equation $-\nabla \cdot(\alpha \nabla u)-u=0$ from 1.1). For this problem, we do not know any analytical solution, and we stress that our goal is not to observe wave propagation nor absorption but to focus on the interface between these regions. In the matrix $\mathrm{A}(5.5)$, only the blocks $A_{1}$ and $A_{2}$ are modified by adding block diagonal terms corresponding to the discretization of $\int_{\Omega_{j}} u_{j}^{N} \overline{v_{j}^{N}}$. The results are displayed in Fig. 4 and can be compared to the results of figures 2 and 3 . We observe that the logarithmic singularity behaviour is present in both illustrations in Fig. 4. Little burr can be seen around the logarithmic singularity, however it seems to be related to the size of the first layer around $\Sigma$ cells and can be expected to resorb as the mesh is refined. 

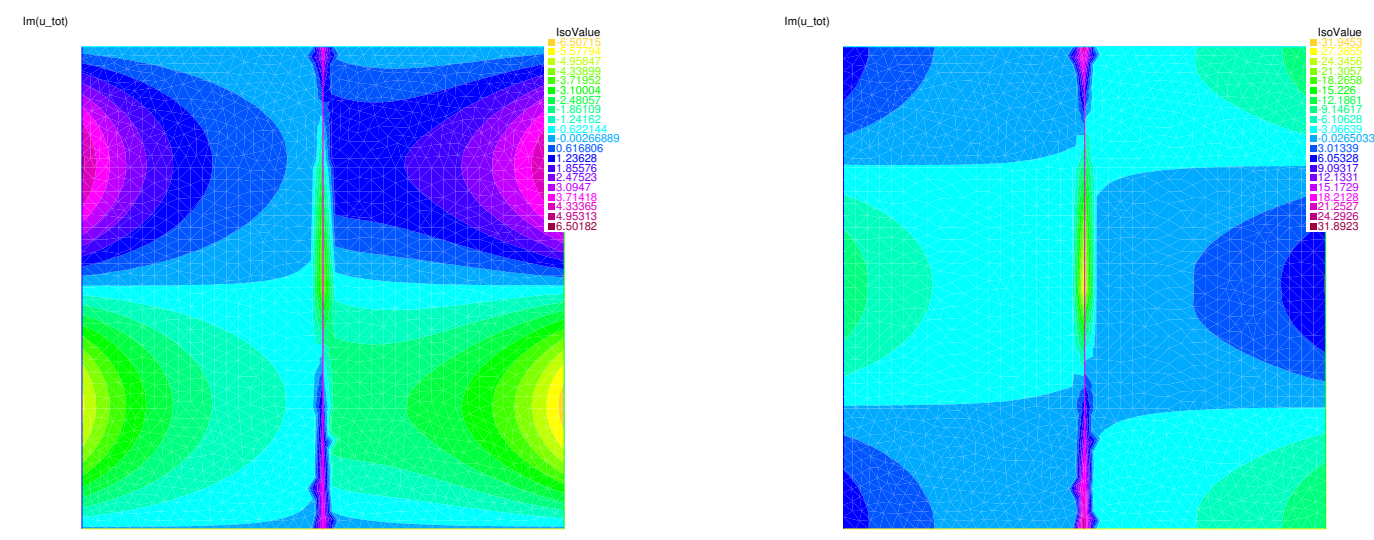

Figure 4: Imaginary parts of the numerical solutions to the complete variational formulation (4.11) with BC $f^{3}$ (left) and $f^{4}$ (right).

\subsubsection{Comments on the value of the penalization parameters}

The numerical illutrations presented above have been obtained with small non-zero values for the penalization parameters 4.10). Non-zero values of the penalization parameters are compatible with the theory presented in this work. Arbitrarily, we used the values $\rho=10^{-2}$ and $\mu=10^{-4}$. However, other simulations taking these parameters equal to 0 lead to results with similar accuracy.

\section{References}

[1] Ammari H., Ciraolo G., Kang H., Lee H. and Milton G. W. (2013) Spectral theory of a NeumannPoincaré-type operator and analysis of cloaking due to anomalous localized resonance. Arch. Rational Mech. Anal., 218, 667-692.

[2] Assous F., Ciarlet Jr. P. and Labrunie S. (2018) Mathematical foundations of computational electromagnetism, Springer-Verlag.

[3] Boffi D., Brezzi F. and Fortin M. (2013) Mixed finite element methods and applications. SpringerVerlag, Berlin.

[4] Bonnet-Ben Dhia A.-S., Chesnel L., Ciarlet Jr. P. (2012) T-coercivity for scalar interface problems between dielectrics and metamaterials. ESAIM: Mathematical Modeling and Numerical Analysis, EDP Sciences, 46, no. 6, 1363-1387.

[5] Bonnet-Ben Dhia A.-S., Chesnel L., Claeys X. (2013) Radiation condition for a non-smooth interface between a dielectric and a metamaterial. M3AS, 23, no. 9, 1629-1662.

[6] Bonnet-Ben Dhia A.-S., Ciarlet Jr. P., Zwölf C. M. (2010) Time harmonic wave diffraction problems in materials with sign-shifting coefficients. Journal of Computational and Applied Mathematics, 234 (2010), 1912-1919. Corrigendum 234, 2616.

[7] Campos-Pinto M. and Després B. 2017 Constructive formulations of resonant Maxwell's equations. SIAM J. Math. Anal. 49, no. 5, 3637-3670.

[8] Ciarlet P. G. (1978) Interpolation error estimates for the reduced Hsieh-Clough-Tocher triangle. Math. of Comp. 32, no. 142, 335-344.

[9] Després B., Imbert-Gérard L.-M.and Weder R. (2014) Hybrid resonance of Maxwell's equations in slab geometry. J. Math. Pures Appl., Elsevier, 101, no. 5, 623-659.

[10] do Carmo M. P. (1976) Differential Geometry of Curves and Surfaces. Prentice-Hall.

[11] Evans L. C. (2010) Partial Differential Equations. American Mathematical Society.

[12] Di Fazio G. , Fanciullo M. S. and Zamboni P. (2008) Harnack inequality and smoothness for quasilinear degenerate elliptic equations, Journal of Differential Equations 245, no. 10, 2939-2957. 
[13] Fabes E. B., Kenig C. E. and Serapioni R. P. 1982 The local regularity of degenerate elliptic equations, Communications in Partial Differential Equations 7, no. 1, 77-116.

[14] Hecht F. (2012) New development in FreeFem++, J. Numer. Math. 20, no. 3-4, 251-265.

[15] Iskhokov S. A., Gadoev M. G. and Konstantinova T. P. 2015 Variational dirichlet problem for degenerate elliptic operators generated by noncoervice forms, Doklady Mathematics 91, no. 3, 255258.

[16] Lu L., Crombé K., Van Eester D., Colas L., Jacquot J. and Heuraux S. (2016) Ion cyclotron wave coupling in the magnetized plasma edge of tokamaks: impact of a finite, inhomogeneous density inside the antenna box. Plasma Physics and Controlled Fusion, 58, no. 5.

[17] Nguyen H.-M. (2016) Limiting absorption principle and well-posedness for the Helmholtz equation with sign changing coefficients. J. Math. Pures Appl., Elsevier, 106, no. 2, 342-374.

[18] Nicolopoulos A. Formulations variationnelles d'équations de Maxwell résonantes, $\mathrm{PhD}$ Thesis, in preparation.

[19] Nicolopoulos A., Campos Pinto M. and Després B. (2019) A stable formulation of resonant Maxwell's equations in cold plasma. Journal of Computational and Applied Mathematics, https://doi.org/10.1016/j.cam.2019.05.007.

[20] Olver F. W. J., Lozier D. W., Boisvert R. F. and Clark C. W. (2010) NIST Handbook of Mathematical Functions. Cambridge University Press, New York.

[21] Stix T. H. (1975) Fast-wave heating of a two-component plasma, Technical Report, Plasma Physics Laboratory, Princeton University.

[22] Triki F. and Vauthrin M. (2018) Mathematical modeling of the photoacoustic effect generated by the heating of metallic nanoparticles. Quarterly of Applied Mathematics, 76, no. 4, 673-698.

[23] Turesson B. O. (2000) Nonlinear Potential Theory and Weighted Sobolev Spaces. Springer-Verlag, Berlin. 


\section{Figure captions}

Figure 2, $\Sigma$ parametrization

Figure 2 Imaginary parts of the exact solution $u^{3}(\mathbf{x})=e^{i \pi y} I_{0}(\pi x)$ (left) and its approximation (right).

Figure 3. Imaginary parts of the exact solution $u^{4}(\mathbf{x})=e^{i \pi y} K_{0}(\pi x)$ (left) and its approximation (right).

Figure 4: Imaginary parts of the numerical solutions to the complete variational formulation (4.11) with $\mathrm{BC} f^{3}$ (left) and $f^{4}$ (right).

\section{Table captions}

Table 1. For the four test cases of (5.2), relative $L^{2}(\Omega)$ error between the exact solution $u_{e x}$ and its approximation $u_{n u m}=u_{j}-w_{g}^{+}$.

Table 2. Residual errors in $L^{2}$ norms for the two first test cases. 